\title{
QUANTUM EXPONENTIAL FUNCTION
}

\author{
S. L. WORONOWICZ* \\ Department of Mathematical Methods in Physics \\ Faculty of Physics, University of Warsaw \\ Hoża 74, 00-682 Warszawa, Poland \\ and \\ Institute of Mathematics, Trondheim University, Norway
}

Received 15 February 1999

\begin{abstract}
A special function playing an essential role in the construction of quantum " $a x+b$ "-group is introduced and investigated. The function is denoted by $F_{\hbar}(r, \varrho)$, where $\hbar$ is a constant such that the deformation parameter $q^{2}=e^{-i \hbar}$. The first variable $r$ runs over non-zero real numbers; the range of the second one depends on the sign of $r: \varrho=0$ for $r>0$ and $\varrho= \pm 1$ for $r<0$. After the holomorphic continuation the function satisfies the functional equation

$$
F_{\hbar}\left(e^{i \hbar} r, \varrho\right)=\left(1+e^{i \hbar / 2} r\right) F_{\hbar}(r,-\varrho) .
$$
\end{abstract}

The name "exponential function" is justified by the formula:

$$
F_{\hbar}(R, \rho) F_{\hbar}(S, \sigma)=F_{\hbar}([R+S], \widetilde{\sigma}),
$$

where $R, S$ are selfadjoint operators satisfying certain commutation relations and $[R+S]$ is a selfadjoint extension of the sum $R+S$ determined by operators $\rho$ and $\sigma$ appearing in the formula. This formula will be used in a forthcoming paper to construct a unitary operator $W$ satisfying the pentagonal equation of Baaj and Skandalis.

\section{Introduction}

Quantum exponential functions appear in the following setting. One considers pairs of closed operators $(R, S)$ acting on a Hilbert space $H$ satisfying certain carefully chosen commutation relations $C$. These relations should imply that the operators $R$ and $S$ are normal and $\operatorname{Sp} R$ and $\operatorname{Sp} S$ be contained in certain subset $\Lambda \subset \mathbf{C}$. Moreover the sum $R+S$ should be a well-defined closed normal operator with the spectrum contained in the same set $\Lambda$. A continuous function $F$ defined on $\Lambda$ with values in the unit circle in $\mathbf{C}$ is called a quantum exponential function if

$$
F(R+S)=F(R) F(S)
$$

for any pair $(R, S)$ of operators satisfying the considered commutation relations.

The first example we obtain considering the following commutation relations:

$$
R^{*}=R, S^{*}=S \text { and } R S=S R .
$$

Then $\Lambda=\mathbf{R}, F$ is a function of real variable and (0.1) is equivalent to the classical exponential equation:

$$
F(r+s)=F(r) F(s)
$$

* Supported by Komitet Badań Naukowych, grant No 2 P0A3 03014.

Reviews in Mathematical Physics, Vol. 12, No. 6 (2000) 873-920

(C) World Scientific Publishing Company 
for any $r, s \in \mathbf{R}$. One of the solutions is $F(r)=e^{i r}$. Any other function satisfying $(0.3)$ is of the form $F_{t}(r)=e^{i t r}$, where $t$ is a real number.

In the second example we consider the following relations:

$$
\left.\begin{array}{c}
R R^{*}=R^{*} R, S S^{*}=S^{*} S, \\
\operatorname{Sp} R, \operatorname{Sp} S \subset \overline{\mathbf{C}}^{q} \text { and } \\
R S=q^{2} S R .
\end{array}\right\}
$$

In these relations $q$ is a real number, $q<1$ and

$$
\overline{\mathbf{C}}^{q}=\left\{t \in \mathbf{C}: t=0 \text { or }|t| \in q^{\mathbf{Z}}\right\} .
$$

The precise meaning of (0.4) is explained in [11] and [12] (such an explanation should also be given for relations (0.2), where $R S=S R$ means the strong commutation of selfadjoint operators $R, S)$. In this case a function $F$ satisfying Eq. (0.1) is given by the formula:

$$
F(t)=\prod_{k=0}^{\infty} \frac{1+q^{2 k} \bar{t}}{1+q^{2 k} t}
$$

Any other function satisfying (0.1) is of the form $F_{t}(r)=F(t r)$, where $t \in \overline{\mathbf{C}}^{q}$.

In this paper we consider the following commutation relations:

$$
\left.\begin{array}{c}
R^{*}=R, S^{*}=S \\
R S=q^{2} S R .
\end{array}\right\}
$$

In this case the deformation parameter $q^{2}$ is a number of modulus 1 . The precise meaning of the relations is given in Sec. 2. We shall assume that

$$
q^{2}=e^{-i \hbar}
$$

where $\hbar$ is a real number such that $-\pi<\hbar<\pi$. This is the only assumption on $\hbar$ imposed in this paper. To construct deformed " $a x+b$ " group [15] we shall have to assume some further restrictions on $q$. It turns out that the group exists on the Hilbert space level (and on the $C^{*}$-level) provided $\hbar= \pm \frac{\pi}{2 k+3}$, where $k=0,1,2, \ldots$. In this case $q$ is a root of 1 .

We write $R \multimap S$ if the pair of selfadjoint operators $(R, S)$ satisfies the relations $(0.5)$ in the sense explained in Sec. 2. The relation "—" is called Zakrzewski relation.

The theory of quantum exponential function based on the relations (0.5) does not fit precisely into the scheme described above. If $R$ and $S$ are selfadjoint operators satisfying the Zakrzewski relation $R \multimap S$, then $R+S$ is a symmetric, but (in general) not a selfadjoint operator. In fact $R+S$ is selfadjoint if and only if $(\operatorname{sign} R)(\operatorname{sign} S) \geq 0$. We shall show that selfadjoint extensions of $R+S$ are labeled by selfadjoint operators $\tau$ such that $\operatorname{Sp} \tau \subset\{-1,0,1\}, \tau$ anticommutes with $R$ and $S$ and $\operatorname{ker} \tau$ coincides with $H((\operatorname{sign} R)(\operatorname{sign} S) \geq 0)$. Operators $\tau$ are called reflection operators.

Let $\rho$ be a selfadjoint operator such that $\operatorname{Sp} \rho \subset\{-1,0,1\}, \rho$ anticommutes with $S$, commutes with $R$ and $\operatorname{ker} \rho=H(R \geq 0)$. Similarly let $\sigma$ a selfadjoint 
operator such that $\operatorname{Sp} \sigma \subset\{-1,0,1\}, \sigma$ anticommutes with $R$, commutes with $S$ and $\operatorname{ker} \sigma=H(S \geq 0)$. Then for any number $\alpha$ of modulus 1 , the operator

$$
\tau=\alpha \rho \sigma+\bar{\alpha} \sigma \rho
$$

satisfies all the conditions listed above. In what follows $\alpha=i e^{\frac{i \pi^{2}}{2 \hbar}}$.

In our case instead of (0.1) we have to consider a more complicated equation

$$
F(R, \rho) F(S, \sigma)=F\left([R+S]_{\tau}, \widetilde{\sigma}\right),
$$

where $[R+S]_{\tau}$ is the selfadjoint extension of $R+S$ corresponding to a reflection operator (0.7) and $\widetilde{\sigma}$ is an operator uniquely determined by $R, S, \rho$ and $\sigma$. The operator $\widetilde{\sigma}$ is selfadjoint, $\operatorname{Sp} \widetilde{\sigma} \subset\{-1,0,1\}, \widetilde{\sigma}$ anticommutes with $[R+S]_{\tau}$ and $\operatorname{ker} \sigma=H\left([R+S]_{\tau} \geq 0\right)$. Now the function $F=F(r, \varrho)$ is a function of two variables: $r \in \mathbf{R}$ and $\varrho \in\{-1,0,1\}$.

The exponential function equality $(0.8)$ is closely related to the pentagonal equation

$$
F(T, \tau) F(S, \sigma) F(R, \rho)=F(R, \rho) F(S, \sigma) .
$$

In the forthcoming paper [15] we shall construct the quantum " $a x+b$ " group. This construction uses the idea of Baaj and Skandalis [2], who pointed out that quantum groups are determined by one unitary operator $W \in B(H \otimes H)$ satisfying certain simple conditions (see also [13]). In [15] we propose an explicit formula for $W$ related to the quantum " $a x+b$ " group. The main aim of the present paper is to provide the computational tools for [15]. In particular (0.9) will be used to verify that $W$ satisfies the Baaj-Skandalis pentagonal equation.

We shall briefly describe the content of the paper. In Sec. 1 we introduce special functions used in this paper. Among them we have the quantum exponential function $F_{\hbar}$ and the related function $V_{\theta}$. Various equalities and estimates are established. Some boring computations are shifted to Appendices. This section is entirely classical: no non-commutative quantity appears in it. We use the methods of the theory of analytic functions of one variable.

The Zakrzewski commutation relations are introduced in Sec. 2. We indicate the connection with the Heisenberg commutation relations. The most general pair $(R, S)$ of selfadjoint operators satisfying the Zakrzewski relations is described.

Section 3 is devoted to operators of the form $S f(R)$. The main result of this section is contained in Theorem 3.1. We find when $S f(R)$ is selfadjoint and compute in this case $\operatorname{sign}(S f(R))$. In particular the operator $e^{i \hbar / 2} R S$ is selfadjoint and $\operatorname{sign}\left(e^{i \hbar / 2} R S\right)=(\operatorname{sign} R)(\operatorname{sign} S)$.

The operator of the form $Q=e^{i \hbar / 2} R S+S$ is investigated in Secs. 4 and 5 . Clearly $Q$ is symmetric. We show that $Q$ is selfadjoint if and only if $R \geq 0$. If this is not the case then we look for selfadjoint extensions. It turns out that selfadjoint extensions of $Q$ are unitarily equivalent to $S$. The correponding unitary operators coincide with $F_{\hbar}(R, \rho)$, where $F_{\hbar}$ is the quantum exponential function and $\rho$ are reflection operators mentioned above:

$$
\left[e^{i \hbar / 2} R S+S\right]_{\rho}=F_{\hbar}(R, \rho)^{*} S F_{\hbar}(R, \rho) .
$$


The main result of the paper is contained in Sec. 6. We prove the exponent equation (0.8) and the pentagonal equation (0.9). To this end we use the method similar to the one used in [11]. In principle it is based on the associativity of addition:

$$
\left[S+e^{i \hbar / 2} S T^{-1}\right]+T^{-1}=S+\left[e^{i \hbar / 2} S T^{-1}+T^{-1}\right] .
$$

The most difficult part of the proof is to show that the formula remains valid, when we pass to suitable selfadjoint extensions:

$$
\left[\left[S+e^{i \hbar / 2} S T^{-1}\right]_{\tau}+T^{-1}\right]_{\widetilde{\sigma}}=\left[S+\left[e^{i \hbar / 2} S T^{-1}+T^{-1}\right]_{\sigma}\right]_{\tilde{\rho}},
$$

where $\widetilde{\rho}=F_{\hbar}(S, \sigma)^{*} \rho F_{\hbar}(S, \sigma)$. Using the unitary implementation (0.10) one can easily show that $(0.11)$ coincides with the most crucial formula (6.11) of the proof of pentagonal equation. The proof of $(0.11)$ is rather complicated. One has to keep track of the domains of all selfadjoint operators appearing in this formula. As a result, Sec. 6 is the most sophisticated in this paper.

In Sec. 7 we show that any function $F$ satisfying the exponential equality $(0.8)$ is of the form: $F(r, \varrho)=F_{\hbar}(\mu r, s \varrho)$. This result will be used to find all unitary representations of quantum " $a x+b$ "-group. The last section deals with $C^{*}$-algebras and affiliation relation. Quantum exponential function is used to formulate a condition for a pair of selfdajoint operators to be affiliated with a $C^{*}$-algebra.

The paper uses heavily the theory of unbounded operators on Hilbert spaces (cf. $[1,4,6,7])$. We shall mainly use closed operators. The domain of an operator $a$ acting on a Hilbert space $H$ will be denoted by $D(a)$. We shall always assume that $D(a)$ is dense in $H$.

We shall use the functional calculus for systems of strongly commuting selfadjoint operators. To explain the rather peculiar but very convenient notation used in the paper, let us consider the pair of strongly commuting selfadjoint operators $a$ and $b$ acting on a Hilbert space $H$. Then, by the spectral theorem

$$
a=\int_{\mathbf{R}^{2}}^{\oplus} \lambda d E(\lambda, \mu), \quad b=\int_{\mathbf{R}^{2}}^{\oplus} \mu d E(\lambda, \mu),
$$

where $d E(\lambda)$ is the common spectral measure associated with $a, b$. For any measurable (complex valued) function $f$ of two variables,

$$
f(a, b)=\int_{\mathbf{R}^{2}}^{\oplus} f\left(\lambda, \lambda^{\prime}\right) d E\left(\lambda, \lambda^{\prime}\right)
$$

Let $\chi$ be the logical evaluation of a sentence:

$$
\begin{aligned}
& \chi(\text { false })=0, \\
& \chi(\text { true })=1 .
\end{aligned}
$$

If $\mathcal{R}$ is a two argument relation defined on real numbers, then $f\left(\lambda, \lambda^{\prime}\right)=\chi\left(\mathcal{R}\left(\lambda, \lambda^{\prime}\right)\right)$ is a characteristic function of the set $\Delta=\left\{\left(\lambda, \lambda^{\prime}\right) \in \mathbf{R}^{2}: \mathcal{R}\left(\lambda, \lambda^{\prime}\right)\right\}$ and (assuming that $\Delta$ is measurable) $f(a, b)=E(\Delta)$. We shall write $\chi(\mathcal{R}(a, b))$ instead of $f(a, b)$ :

$$
\chi(\mathcal{R}(a, b))=\int_{\mathbf{R}^{2}}^{\oplus} \chi\left(\mathcal{R}\left(\lambda, \lambda^{\prime}\right)\right) d E\left(\lambda, \lambda^{\prime}\right)=E(\Delta) .
$$


The range of this projection will be denoted by $H(\mathcal{R}(a, b))$. The letter " $H$ " in this expression refers to the Hilbert space, where operators $a, b$ act.

This way we gave meaning to the expressions: $\chi(a>b), \chi\left(a^{2}+b^{2}=1\right), \chi(a=1)$, $\chi(b<0), \chi(a \neq 0)$ and many others of this form. They are orthogonal projections onto corresponding spectral subspaces. For example $H(a=1)$ is the eigenspace of $a$ corresponding to the eigenvalue 1 and $\chi(a=1)$ is the orthogonal projection onto this eigenspace. More generally if $\Delta$ is a measurable subset of $\mathbf{R}$, then $H(a \in \Delta)$ is the spectral subspace of $a$ corresponding to $\Delta$ and $\chi(a \in \Delta)$ is the corresponding spectral projection.

\section{Special Functions}

The quantum exponential function is introduced in an axiomatic way. In this section we formulate the conditions determining uniquely this function. The function is then defined by an explicit formula and we verify that all the conditions hold.

Let $\mathbf{C}_{*}=\mathbf{C}-\{0\}$. The following domains in $\mathbf{C}$ will play an essential role in this paper. For $\hbar>0$ we set

$$
\left.\begin{array}{l}
\Omega_{\hbar}^{+}=\left\{r \in \mathbf{C}_{*}: \arg r \in[0, \hbar]\right\} \\
\Omega_{\hbar}^{-}=\left\{r \in \mathbf{C}_{*}: \arg r \in[-\pi, \hbar-\pi]\right\} \\
\Omega_{\hbar}=\Omega_{\hbar}^{-} \cup \Omega_{\hbar}^{+} .
\end{array}\right\}
$$

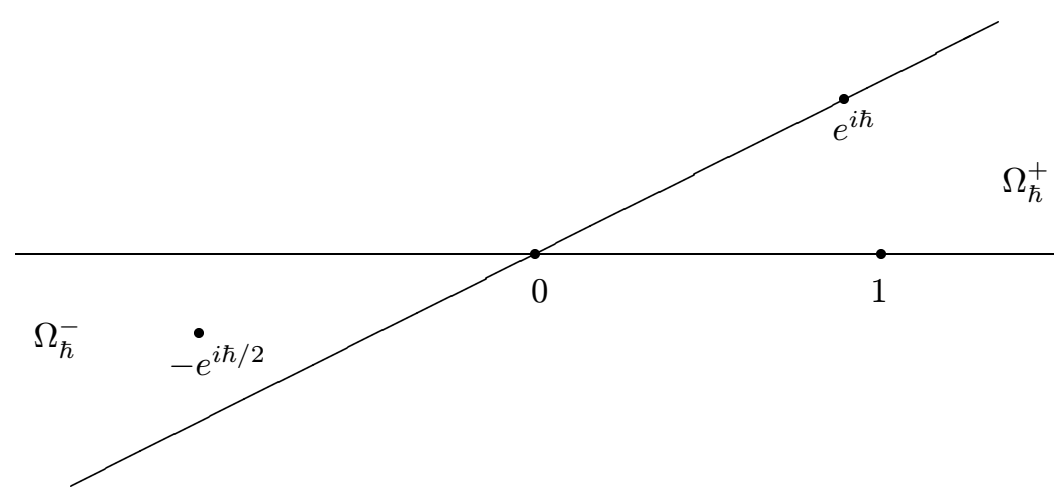

For $\hbar<0$, in the above formulae one has to replace $[0, \hbar]$ and $[-\pi, \hbar-\pi]$ by $[\hbar, 0]$ and $[\hbar-\pi,-\pi]$. For any $r \in \Omega_{\hbar}$ we set

$$
\ell(r)=\left\{\begin{array}{ll}
\log |r|+i \arg r & \text { for } r \in \Omega_{\hbar}^{+} \\
\log |r|+i(\arg r+\pi) & \text { for } r \in \Omega_{\hbar}^{-}
\end{array},\right.
$$

where $\arg r$ is taken from the range indicated in (1.1). Clearly the function $\ell$ is continuous on $\Omega_{\hbar}$ and holomorphic on the interior of this set. Let

$$
\mathcal{H}=\left\{\begin{array}{cc}
f \text { is holomorphic on the interior of } \Omega_{\hbar} \text { and } \\
f \in C\left(\Omega_{\hbar}\right): & \text { for any } \lambda>0 \text { the function } e^{-\lambda \ell(r)^{2}} f(r) \\
\text { is bounded on } \Omega_{\hbar}
\end{array}\right\} .
$$


Replacing in this definition $\Omega_{\hbar}$ by $\Omega_{\hbar}^{+}$and $\Omega_{\hbar}^{-}$we introduce the classes of functions $\mathcal{H}^{+}$and $\mathcal{H}^{-}$.

The vector space $\mathcal{H}$ (as well as $\mathcal{H}^{+}$and $\mathcal{H}^{-}$) admits an interesting antiunitary involution. For any $f \in \mathcal{H}$ and $r \in \Omega_{\hbar}$ we set

$$
f^{*}(r)=\overline{f\left(e^{i \hbar} \bar{r}\right)} .
$$

One can easily verify that $f^{*} \in \mathcal{H}$ and $f^{* *}=f$.

The quantum exponential function $F_{\hbar}$ investigated in this paper is a function of two variables denoted by $r$ and $\varrho$. The first one runs over $\Omega_{\hbar}$ whereas the second assumes three values: $-1,0,1$ with the following restrictions: $\varrho=0$ for $r \in \Omega_{\hbar}^{+}$and $\varrho= \pm 1$ for $r \in \Omega_{\hbar}^{-}$. In other words, $F_{\hbar}$ is defined on the set

$$
\Delta_{F}=\Omega_{\hbar}^{-} \times\{-1,1\} \cup \Omega_{\hbar}^{+} \times\{0\} .
$$

In the following theorem we collect the characteristic properties of $F_{\hbar}$.

Theorem 1.1. There exists a unique function

$$
F_{\hbar}: \Delta_{F} \longrightarrow \mathbf{C}
$$

fulfilling the following five conditions:

(1) For all $(r, \varrho) \in \Delta_{F}$ such that $r \in \mathbf{R}$ we have:

$$
\left|F_{\hbar}(r, \varrho)\right|=1 .
$$

(2) The functions $F_{\hbar}(\cdot, 0), F_{\hbar}(\cdot, 0)^{-1} \in \mathcal{H}^{+}$.

(3) The functions $F_{\hbar}(\cdot, 1), F_{\hbar}(\cdot,-1), F_{\hbar}(\cdot,-1)^{-1} \in \mathcal{H}^{-}$.

(4) For any $(r, \varrho) \in \Delta_{F}$ such that $r \in \mathbf{R}$ we have:

$$
F_{\hbar}\left(e^{i \hbar} r, \varrho\right)=\left(1+e^{i \hbar / 2} r\right) F_{\hbar}(r,-\varrho) .
$$

$(5)$

$$
\lim _{r \rightarrow 0} F_{\hbar}(r, \varrho)=1 .
$$

In addition the function $F_{\hbar}$ has the following properties:

(6) $F_{\hbar}\left(-e^{i \hbar / 2}, 1\right)=0$. This is the only zero of $F_{\hbar}$ on $\Delta_{F}$.

(7) In a neighbourhood of zero,

$$
F_{\hbar}(r, \varrho)=1+\frac{r}{2 i \sin \frac{\hbar}{2}}+\mathcal{R}_{o}(r, \varrho),
$$

where

$$
\lim _{r \rightarrow 0} \frac{\mathcal{R}_{o}(r, \varrho)}{r}=0 .
$$

This is a stronger version of Statement (5).

(8) There exists the limit

$$
\lim _{\Re r \rightarrow+\infty} \frac{F_{\hbar}(-r, 1)}{F_{\hbar}(r, 0)}=\alpha,
$$


where

$$
\alpha=i \exp \left\{\frac{i \pi^{2}}{2 \hbar}\right\} .
$$

For real $r$ we have a stronger result:

$$
\lim _{\substack{r-\text { real } \\ r \rightarrow+\infty}}\left|r\left(F_{\hbar}(-r, 1)-\alpha F_{\hbar}(r, 0)\right)\right|=\frac{1}{\sin \frac{\hbar}{2}} .
$$

(9) For any $r \in \Omega_{\hbar}^{+}$we have

$$
F_{\hbar}(r, 0)=C \exp \left\{\frac{(\log r)^{2}}{2 i \hbar}\right\} \overline{F_{\hbar}\left(\bar{r}^{-1}, 0\right)} .
$$

In a neighbourhood of infinity

$$
F_{\hbar}(r, 0)=C \exp \left\{\frac{(\log r)^{2}}{2 i \hbar}\right\}\left[1-\frac{r^{-1}}{2 i \sin \frac{\hbar}{2}}\right]+\mathcal{R}_{\infty}^{+}(r),
$$

where

$$
\begin{aligned}
& \lim _{\Re r \rightarrow+\infty} \mathcal{R}_{\infty}^{+}(r)=0, \\
& \lim _{\substack{r \text {-real } \\
r \rightarrow+\infty}} r \mathcal{R}_{\infty}^{+}(r)=0 .
\end{aligned}
$$

(10) For any $r \in \Omega_{\hbar}^{-}$and $\varrho= \pm 1$ we have

$$
F_{\hbar}(r, \varrho)=C \alpha \varrho \exp \left\{\frac{(\log (-r))^{2}}{2 i \hbar}\right\} \overline{F_{\hbar}\left(\bar{r}^{-1}, \varrho\right)} .
$$

In a neighbourhood of infinity

$$
F_{\hbar}(r, \varrho)=C \alpha \varrho \exp \left\{\frac{(\log (-r))^{2}}{2 i \hbar}\right\}\left[1-\frac{r^{-1}}{2 i \sin \frac{\hbar}{2}}\right]+\mathcal{R}_{\infty}^{-}(r, \varrho),
$$

where

$$
\begin{aligned}
& \lim _{\Re r \rightarrow-\infty} \mathcal{R}_{\infty}^{-}(r, \varrho)=0, \\
& \lim _{\substack{r \text {-real } \\
r \rightarrow-\infty}} r \mathcal{R}_{\infty}^{-}(r, \varrho)=0 .
\end{aligned}
$$

In the last two points, the constant $C=\exp \left\{\left(\frac{2 \pi}{\hbar}+\frac{\hbar}{2 \pi}\right) \frac{\pi}{12 i}\right\}, \log r=\log |r|+i \arg r$ and $\log (-r)=\log |r|+i(\pi+\arg r)=\log r+i \pi$, where $\arg r$ is taken from the range indicated in (1.1).

In this section we find an explicit formula expressing $F_{\hbar}(r, \varrho)$ in terms of integrals of elementary functions. We shall prove that $F_{\hbar}(r, \varrho)$ has the properties listed in the above theorem. The uniqueness will be discussed later (cf. the end of Sec. 5). 
The reader should notice that if a function $F_{\hbar}(r, \varrho)$ satisfies any of the conditions $(1)-(9)$ of the above theorem then the function $F_{\hbar}^{\prime}(r, \varrho)=\overline{F_{\hbar}(\bar{r}, \varrho)}$ satisfies the same conditions with $\hbar$ replaced by $-\hbar$. It means that

$$
F_{-\hbar}(r, \varrho)=\overline{F_{\hbar}(\bar{r}, \varrho)}
$$

Due to this formula it is sufficient to consider the case $\hbar>0$.

To simplify the notation we set $\theta=\frac{2 \pi}{\hbar}$. Clearly $\theta>2$. For any $(r, \varrho) \in \Delta_{F}$ we have

$$
F_{\hbar}(r, \varrho)=\left[1+i \varrho(-r)^{\frac{\pi}{\hbar}}\right] V_{\theta}(\log r)
$$

In this formula $\log r=\log |r|+i \arg r$ and $(-r)^{\frac{\pi}{\hbar}}=|r|^{\frac{\pi}{\hbar}} \exp \left(\frac{i \pi}{\hbar}[\pi+\arg r]\right)$, where $\arg r$ is taken from the range indicated in (1.1). $V_{\theta}$ is a meromorphic function on C such that

$$
V_{\theta}(x)=\exp \left\{\frac{1}{2 \pi i} \int_{0}^{\infty} \log \left(1+a^{-\theta}\right) \frac{d a}{a+e^{-x}}\right\}
$$

for all $x \in \mathbf{C}$ such that $|\Im x|<\pi$. For real $r$ we have:

$$
F_{\hbar}(r, \varrho)=\left\{\begin{array}{lll}
V_{\theta}(\log r) & \text { for } \quad r>0 \text { and } \varrho=0 \\
{\left[1+i \varrho|r|^{\frac{\pi}{\hbar}}\right] V_{\theta}(\log |r|-\pi i)} & \text { for } \quad r<0 \text { and } \varrho= \pm 1
\end{array}\right.
$$

Lemma 1.1. Let $\theta$ be a positive number. Then the integral

$$
W_{\theta}(x)=\int_{0}^{\infty} \log \left(1+a^{-\theta}\right) \frac{d a}{a+e^{-x}}
$$

is convergent for any $x \in \mathbf{C}$ such that $|\Im x|<\pi$. The function $W_{\theta}$ introduced in this way is holomorphic in the strip $\{x \in \mathbf{C}:|\Im x|<\pi\}$. It admits a continuous extension to the closure of this strip. Moreover we have:

$$
\begin{gathered}
W_{\theta}(x)+W_{\theta}(-x)=\frac{\theta x^{2}}{2}+\left(\theta+\theta^{-1}\right) \frac{\pi^{2}}{6}, \\
W_{1 / \theta}(x)=W_{\theta}(x / \theta), \\
W_{\theta}(x+\pi i)-W_{\theta}(x-\pi i)=2 \pi i \log \left(1+e^{\theta x}\right) .
\end{gathered}
$$

In (1.23) the variable $x$ is real, whereas in (1.21) and (1.22) $x \in \mathbf{C}$ and $|\Im x| \leq \pi$. If $\theta>2$, then

$$
W_{\theta}(x)=\frac{\pi e^{x}}{\sin \left(\frac{\pi}{\theta}\right)}-\frac{\pi e^{2 x}}{2 \sin \left(\frac{2 \pi}{\theta}\right)}+\mathcal{R}(x)
$$

where

$$
e^{-2 x} \mathcal{R}(x) \longrightarrow 0
$$

when $\Re x \rightarrow-\infty$ whereas $\Im x$ stays in a compact subset of the interval $]-\pi, \pi[$.

The proof of this lemma is not very fascinating. It uses the standard methods of the theory of holomorphic functions of one variable. The boring details are shifted to Appendix A. 
Comparing (1.18) with (1.20) we see that

$$
V_{\theta}(x)=\exp \left\{\frac{1}{2 \pi i} W_{\theta}(x)\right\} .
$$

Taking into account (1.22) and (1.23) we obtain:

$$
\begin{gathered}
V_{1 / \theta}(x)=V_{\theta}(x / \theta), \\
V_{\theta}(x+\pi i)=\left(1+e^{\theta x}\right) V_{\theta}(x-\pi i) .
\end{gathered}
$$

Inserting in (1.28) $x / \theta$ instead of $x$ and using (1.27) we obtain

$$
V_{1 / \theta}(x+\pi \theta i)=\left(1+e^{x}\right) V_{1 / \theta}(x-\pi \theta i) .
$$

Replacing now $1 / \theta$ by $\theta$ we get

$$
V_{\theta}\left(x+\frac{\pi i}{\theta}\right)=\left(1+e^{x}\right) V_{\theta}\left(x-\frac{\pi i}{\theta}\right) .
$$

Remark 1.1. Equation (1.28) is essentially the same as functional equation defining function $s$ in [3].

According to (1.26), the function $V_{\theta}$ is holomorphic and has no zeroes in the strip $\{x \in \mathbf{C}:|\Im x|<\pi\}$. Due to (1.28), it admits an analytical continuation to a meromorphic function defined on the whole plane $\mathbf{C}$. The extended function has zeroes at the points

$$
x=(2 k+1) \pi i+(2 l+1) \pi i / \theta,
$$

where $k, l=0,1,2, \ldots$ The poles are located at points $-x$, where $x$ is given by (1.30). Multiplicity of the zero (the pole respectively) at the point $x(-x$ respectively) equals to the number of pairs $(k, l)$ of nonnegative integers satisfying the relation (1.30). If $\theta$ is irrational, then all zeroes and poles are simple.

Replacing in (1.29) $x$ by $x+\frac{\pi i}{\theta}$ we obtain

$$
V_{\theta}\left(x+\frac{2 \pi i}{\theta}\right)=\left(1+e^{\pi i / \theta} e^{x}\right) V_{\theta}(x)
$$

Taking into account (1.21) we get

$$
V_{\theta}(x) V_{\theta}(-x)=C_{\theta} \exp \left\{\frac{\theta x^{2}}{4 \pi i}\right\},
$$

where $C_{\theta}=\exp \left\{\left(\theta+\theta^{-1}\right) \frac{\pi}{12 i}\right\}$. Clearly $W_{\theta}(x)$ is real for $x$-real. Therefore

$$
\left|V_{\theta}(x)\right|=1
$$

for all $x \in \mathbf{R}$. Taking the square of the both sides and performing analytical continuation we get

$$
\overline{V_{\theta}(\bar{x})} V_{\theta}(x)=1
$$


and $\left|V_{\theta}(\bar{x})\right|\left|V_{\theta}(x)\right|=1$. In particular $\left|V_{\theta}(x+\pi i)\right|\left|V_{\theta}(x-\pi i)\right|=1$ for all $x \in \mathbf{R}$. On the other hand, due to (1.28) $\left|V_{\theta}(x+\pi i)\right|=\left|1+e^{\theta x}\right|\left|V_{\theta}(x-\pi i)\right|$. Therefore

$$
\left|V_{\theta}(x-\pi i)\right|=\left|1+e^{\theta x}\right|^{-\frac{1}{2}}
$$

for any $x \in \mathbf{R}$. Combining (1.34) with (1.32) we obtain

$$
V_{\theta}(x)=C_{\theta} \exp \left\{\frac{\theta x^{2}}{4 \pi i}\right\} \overline{V_{\theta}(-\bar{x})}
$$

for all $x \in \mathbf{C}$.

In further computations we set $\theta=\frac{2 \pi}{\hbar}$, where $0<\hbar<\pi$. Then $\theta>2$ and using (1.24) and (1.25) we get

$$
V_{\frac{2 \pi}{\hbar}}(x)=1+\frac{e^{x}}{2 i \sin \frac{\hbar}{2}}-\frac{e^{2 x-i \hbar / 2}}{4 \sin \hbar \sin \frac{\hbar}{2}}+\mathcal{R}_{1}(x),
$$

where

$$
e^{-2 x} \mathcal{R}_{1}(x) \longrightarrow 0,
$$

when $\Re x \rightarrow-\infty$ whereas $|\Im x| \leq \hbar / 2$. It is enough to assume that $(\Im x) e^{\Re x}$ stays bounded. Indeed, replacing in (1.31) $\theta$ by $\frac{2 \pi}{\hbar}$ and inserting (1.37) instead of $V_{\theta}(x)$ we get after simple computations:

$$
\mathcal{R}_{1}(x+i \hbar)=\left(1+e^{i \hbar / 2} e^{x}\right) \mathcal{R}_{1}(x) .
$$

Let $\omega$ be a product of $n$ factors of the form $1+e^{x}$ with the same $\Re x<0$. Elementary computations show that

$$
\exp \left\{-\frac{n e^{\Re x}}{1-e^{\Re x}}\right\} \leq|\omega| \leq \exp \left\{n e^{\Re x}\right\} .
$$

Now, taking into account (1.39) can easily show that (1.38) holds when $\Re x \rightarrow-\infty$ whereas $(\Im x) e^{\Re x}$ stays bounded. In particular

$$
\lim _{t \rightarrow+\infty} V_{\theta}\left(x_{o}+t \lambda\right)=1
$$

for any $x_{o}, \lambda \in \mathbf{C}$ such that $\Im \lambda<0$.

To prove the manageability of the multiplicative unitary for " $a x+b$ " quantum group [15] we have to compute the Fourier transform of $V_{\theta}$. It turns out that it is expressed by the same function. Setting $\theta=\frac{2 \pi}{\hbar}$ we have

$$
\frac{1}{\sqrt{2 \pi \hbar}} \int_{\ell} V_{\theta}\left(y-\frac{i \hbar}{2}-i \pi\right) e^{\frac{i y^{2}}{2 \hbar}} e^{\frac{i x y}{\hbar}} d y=C_{\hbar}^{\prime} V_{\theta}(x) .
$$

In this formula $C_{\hbar}^{\prime}$ is the phase factor equal to $\exp \left\{i\left(\frac{\pi}{4}+\frac{\hbar}{24}+\frac{\pi^{2}}{6 \hbar}\right)\right\}$, the path $\ell$ goes along the real axis from $-\infty$ to $\infty$ rounding the pole of the integrand at the point 0 from above. The integral should be understood in the sense of the distribution theory; before comparing the numerical values of the both sides one has to multiply 
them by a test function of $x$ and integrate with respect to $x$ (on the left-hand side the integral with respect to $y$ should be taken after that with respect to $x$ ). The proof of (1.41) is given in Appendix B.

Now we are able to show that the function (1.17) satisfies conditions (1)-(10) of Theorem 1.1. Condition (1) follows from (1.33) and (1.35). For $r>0$ formula (1.6) coincides with (1.31). To prove it for $r<0$ it is sufficient to notice that $(-r)^{\frac{\pi}{\hbar}}$ changes sign, when $\arg r$ increases by $\hbar$. Condition (4) is verified. Conditions (7) and (5) follows immediately from (1.37).

Inserting in (1.36) $\theta=2 \pi / \hbar$ we obtain (1.12). Combining (1.12) with (1.8) we get (1.13), with

$$
\mathcal{R}_{\infty}^{+}(r)=C \exp \left\{\frac{(\log r)^{2}}{2 i \hbar}\right\} \mathcal{R}_{o}(r, 0)
$$

Therefore

$$
\left|\mathcal{R}_{\infty}^{+}(r)\right|=|r|^{\frac{\arg r}{\hbar}}\left|\mathcal{R}_{o}(r, 0)\right|
$$

and Condition (7) assures the correct asymptotic behavior of $\mathcal{R}_{\infty}^{+}(r)$. Condition (9) is verified.

To prove Condition (10) assume that $r \in \Omega_{\hbar}^{-}$and $\varrho= \pm 1$. Then

$$
\begin{aligned}
\frac{F_{\hbar}(r, \varrho)}{\overline{F_{\hbar}\left(\bar{r}^{-1}, \varrho\right)}} & =\frac{V_{\frac{2 \pi}{\hbar}}(\log r)\left[1+i \varrho(-r)^{\frac{\pi}{\hbar}}\right]}{\overline{V_{\frac{2 \pi}{\hbar}}(-\overline{\log r})\left[1+i \varrho(-\bar{r})^{-\frac{\pi}{\hbar}}\right]}} \\
& =C_{\frac{2 \pi}{\hbar}} \exp \left\{\frac{(\log r)^{2}}{2 i \hbar}\right\} \frac{1+i \varrho(-r)^{\frac{\pi}{\hbar}}}{1-i \varrho(-r)^{-\frac{\pi}{\hbar}}} \\
& =C_{\frac{2 \pi}{\hbar}} \exp \left\{\frac{(\log r)^{2}}{2 i \hbar}\right\} i \varrho(-r)^{\frac{\pi}{\hbar}} \\
& =i \varrho C_{\frac{2 \pi}{\hbar}} \exp \left\{\frac{(\log r)^{2}+2 \pi i \log (-r)}{2 i \hbar}\right\} .
\end{aligned}
$$

In second step of the above computations we used (1.36). Remembering that $\log (-r)=\log r+i \pi$ we obtain

$$
\frac{F_{\hbar}(r, \varrho)}{\overline{F_{\hbar}\left(\bar{r}^{-1}, \varrho\right)}}=i \varrho C_{\frac{2 \pi}{\hbar}} \exp \left\{\frac{(\log (-r))^{2}}{2 i \hbar}+\frac{i \pi^{2}}{2 \hbar}\right\}=\varrho \alpha C_{\frac{2 \pi}{\hbar}} \exp \left\{\frac{(\log (-r))^{2}}{2 i \hbar}\right\},
$$

where $\alpha$ is given by (1.10). Formula (1.14) is verified. Combining this formula with (1.8) we obtain (1.15). Condition (10) is verified.

To verify Condition (8) we have to compute the limits (1.9) and (1.11). Using asymptotic formulae (1.13) and (1.15) one can easily show that the limits exist and have the correct values.

Definition (1.26) shows that $V_{\frac{2 \pi}{\hbar}}(x) \neq 0$ for all $x \in \mathbf{C}$ such that $|\Im x| \leq \pi$. Therefore $F_{\hbar}(r, \varrho)=0$ if and only if $r \in \Omega_{\hbar}^{-}, \varrho= \pm 1$ and $1+(-r)^{\frac{\pi}{\hbar}}=0$. One can easily check that this is the case if and only if $r=-e^{i \hbar / 2}$ and $\varrho=1$. Condition (6) is verified. 
Finally using (1.7) and the asymptotic behavior (1.13) and (1.15) and remembering that $F_{\hbar}(\cdot, 0)$ has no zero in $\Omega_{\hbar}^{+}$and $F_{\hbar}(\cdot,-1)$ has no zero in $\Omega_{\hbar}^{-}$one can easily show that $F_{\hbar}(\cdot, 0), F_{\hbar}(\cdot, 0)^{-1} \in \mathcal{H}^{+}$and $F_{\hbar}(\cdot, 1), F_{\hbar}(\cdot,-1), F_{\hbar}(\cdot,-1)^{-1} \in \mathcal{H}^{-}$. The reader should notice that $F_{\hbar}(\cdot, 1)^{-1} \notin \mathcal{H}^{-}$, because the function $F_{\hbar}(\cdot, 1)$ has a zero point in $\Omega_{\hbar}^{-}$. Conditions (2) and (3) are verified.

\section{Zakrzewski Commutation Relations}

Let $R$ and $S$ be selfadjoint operators acting on a Hilbert space $H$. If $\operatorname{sign} R$ commutes with $S$ and $R$ commutes with $\operatorname{sign} S$, then

$$
H=H_{00} \oplus H_{0 S} \oplus H_{R 0} \oplus H_{R S},
$$

where $H_{00}=\operatorname{ker} R \cap \operatorname{ker} S, H_{0 S}=\operatorname{ker} R \cap(\operatorname{ker} S)^{\perp}, H_{R 0}=(\operatorname{ker} R)^{\perp} \cap \operatorname{ker} S$ and $H_{R S}=(\operatorname{ker} R)^{\perp} \cap(\operatorname{ker} S)^{\perp}$. Clearly operators $R$ and $S$ respect the above direct sum decomposition and $|R|$ and $|S|$ are selfadjoint, strictly positive on $H_{R S}$. Therefore one can consider one parameter groups of unitaries $\left(|R|^{i \tau}\right)_{\tau \in \mathbf{R}}$ and $\left(|S|^{i \tau}\right)_{\tau \in \mathbf{R}}$ acting on $H_{R S}$.

Definition 2.1. We say that the operators $R$ and $S$ satisfy the Zakrzewski commutation relations and write $R \multimap S$ if:

(1) $\operatorname{sign} R$ commutes with $S$ and $R$ commutes with $\operatorname{sign} S$,

(2) On the subspace $H_{R S}=(\operatorname{ker} R)^{\perp} \cap(\operatorname{ker} S)^{\perp}$, the groups of unitaries satisfy the canonical commutation relations in the Weyl form:

$$
|R|^{i \tau}|S|^{i \tau^{\prime}}=e^{i \hbar \tau \tau^{\prime}}|S|^{i \tau^{\prime}}|R|^{i \tau}
$$

for any $\tau, \tau^{\prime} \in \mathbf{R}$. The pair is called non-degenerate if $\operatorname{ker} R=\operatorname{ker} S=\{0\}$. This is the case if and only if $H_{R S}=H$.

One can easily check that $R \multimap S$ if and only if $R$ commutes with $\operatorname{sign} S$ and for any $\tau \in \mathbf{R}$

$$
|S|^{-i \tau} R|S|^{i \tau}=e^{\hbar \tau} R \text {. }
$$

The latter equation should hold on the subspace $(\operatorname{ker} S)^{\perp}$.

Similarly $R \multimap S$ if and only if $S$ commutes with $\operatorname{sign} R$ and for any $\tau \in \mathbf{R}$

$$
|R|^{i \tau} S|R|^{-i \tau}=e^{\hbar \tau} S
$$

The latter equation should hold on the subspace $(\operatorname{ker} R)^{\perp}$.

Let $R$ and $S$ be selfadjoint operators acting on a Hilbert space $H$. Assume that $R \multimap S$. Then the compositions $R \circ S$ and $S \circ R$ are densely defined closeable operators, $D(R \circ S)$ is a core for $S$ and $D(S \circ R)$ is a core for $R$. In what follows, the closures of $R \circ S$ and $S \circ R$ will be denoted by $R S$ and $S R$. It turns out that $R$ and $S$ satisfy the commutation relation of the Manin plane: $D(R S)=D(S R)$ and

$$
R S x=q^{2} S R x
$$


for any $x \in D(S R)$. The reader should notice that the condition (2.5) is much weaker than the relation $R \multimap S$. For example (2.5) remains unchanged, when $\hbar$ is replaced by $\hbar+2 \pi$, whereas (2.2) is very sensitive to the choice of $\hbar$ solving Eq. (0.6).

We shall use the following simple statement:

Proposition 2.1. Let $R$ and $S$ be selfadjoint operators acting on a Hilbert space $H$. Assume that $R \multimap S$. Then:

(1) If $\operatorname{ker} S=\{0\}$, then $S^{-1} \multimap R$.

(2) If $\operatorname{ker} R=\{0\}$, then $S \multimap R^{-1}$.

One can easily describe the structure of Hilbert space given by a pair $(R, S)$ of selfadjoint operators satisfying the relation $R \multimap S$. We already know that the operators $R$ and $S$ respect the decomposition (2.1). On $H_{00}$ both operators vanish. On $H_{R 0}$ the operator $S$ vanishes, whereas $R$ is any selfadjoint operator. Similarly on $H_{0 S}, R$ vanishes whereas $S$ is any selfadjoint operator (if one of the operators vanish then the relation imposes no condition on the other one). On $H_{R S}$ the pair is non-degenerate.

To describe nondegenerate pairs $(R, S)$ of selfadjoint operators satisfying the Zakrzewski relation we shall use position and momentum operators of a quantum mechanical particle moving on $\mathbf{R}$. Let $(\hat{q}, \hat{p})$ be an irreducible pair of selfadjoint operators acting on a Hilbert space $L$ such that the Heisenberg-Weyl commutation relations:

$$
e^{i \tau \hat{q}} e^{i \tau^{\prime} \hat{p}}=e^{i \hbar \tau \tau^{\prime}} e^{i \tau^{\prime} \hat{p}} e^{i \tau \hat{q}}
$$

are satisfied for all $\tau, \tau^{\prime} \in \mathbf{R}$. In short $[\hat{p}, \hat{q}]=i \hbar I$. Irreducibility means that the only operators on $L$ commuting with $e^{i \tau \hat{q}}$ and $e^{i \tau^{\prime} \hat{p}}$ are scalar multiples of $I$. By the famous Stone-von Neumann theorem [9], the pair $(\hat{q}, \hat{p})$ is unique up to unitary equivalence. In Schrödinger representation $L=L^{2}(\mathbf{R})$ and

$$
(\hat{q} \widetilde{x})(t)=t \widetilde{x}(t), \quad(\hat{p} \widetilde{x})(t)=i \hbar \frac{d \widetilde{x}(t)}{d t} .
$$

By definition a square integrable function $\widetilde{x}$ belongs to $D(\hat{q})$ iff the product $t \widetilde{x}(t)$ is square-integrable. Similarly $\widetilde{x} \in D(\hat{p})$ iff the derivative (understood in the sense of the distribution theory) $\frac{d \widetilde{x}(t)}{d t}$ is a square-integrable function. In Sec. 4 we shall use another representation: $L=L^{2}\left(\mathbf{R}_{+}\right)$and

$$
(\hat{q} x)(s)=i \hbar\left[-\frac{x(s)}{2}+\frac{d}{d s}(s x(s))\right], \quad(\hat{p} x)(s)=-(\log s) x(s) .
$$

Now a square integrable function $x$ belongs to $D(\hat{q})(x \in D(\hat{p})$ respectively) iff the function $\frac{d}{d s}(s x(s))\left((\log s) x(s)\right.$ respectively) is square integrable on $\mathbf{R}_{+}$. The two representations are related by the unitary Mellin transform:

$$
x(s)=\frac{1}{\sqrt{2 \pi|\hbar| s}} \int_{\mathbf{R}} s^{-\frac{i t}{\hbar}} \widetilde{x}(t) d t .
$$

With the notation introduced above we have: 
Theorem 2.1. Let $u$ and $v$ be two commuting unitary involutions acting on a Hilbert space $K: u=u^{*}=u^{-1}, v=v^{*}=v^{-1}$ and $u v=v u$. Then the pair $(R, S)=\left(u \otimes e^{\hat{q}}, v \otimes e^{\hat{p}}\right)$ is a non-degenerate pair of selfadjoint operators acting on $L^{2}(\mathbf{R}, K)=K \otimes L^{2}(\mathbf{R})$ and $R \multimap S$. Conversely any nondegenerate pair $(R, S)$ of selfadjoint operators satisfying the Zakrzewski relation $R \multimap S$ is unitarily equivalent to a pair $\left(u \otimes e^{\hat{q}}, v \otimes e^{\hat{p}}\right)$ described above.

Proof. Only the last statement needs a proof. Let $(R, S)$ be a non-degenerate pair of selfadjoint operators acting on a Hilbert space $H$ and $R \multimap S$. Then the operators $\log |R|$ and $\log |S|$ are selfadjoint and satisfy the Heisenberg commutation relation in the Weyl form. By the Stone-von Neumann theorem [9] the pair $(\log |R|, \log |S|)$ is unitarily equivalent to the direct sum of a certain number of copies of $(\hat{q}, \hat{p})$. Denoting the multiplicity by $N$ we may identify $H$ with $K \otimes L$ (where $K$ is an $N$-dimensional Hilbert space) in such a way that

$$
\log R=I \otimes \hat{q}, \quad \log S=I \otimes \hat{p} .
$$

Irreducibility of $(\hat{q}, \hat{p})$ implies that any operator $Q$ commuting with $\hat{q}$ and $\hat{p}$ is of the form $Q=Q_{o} \otimes I$, where $Q_{o}$ is an operator acting on $K$. In particular

$$
\operatorname{sign} R=u \otimes I, \quad \operatorname{sign} S=v \otimes I,
$$

where $u$ and $v$ are commuting unitary involutions acting on $K$. Combining (2.8) with (2.9) we obtain

$$
R=u \otimes e^{\hat{q}}, \quad S=v \otimes e^{\hat{p}} .
$$

The number $N=\operatorname{dim} K$ will be called the multiplicity of the pair $(R, S)$. Clearly

$$
N=N_{++}+N_{+-}+N_{-+}+N_{--}
$$

where $N_{ \pm \pm}=\operatorname{dim} K(u= \pm 1, v= \pm 1)$. Two nondegenerate pairs of selfadjoint operators satisfying the Zakrzewski relation are unitarily equivalent iff they have the same numbers $N_{ \pm \pm}$.

\section{Analytical Implications of the Zakrzewski Relation}

The selfadjoint operators satisfying the Zakrzewski relation have interesting analytical properties. On the formal level the relation $R S=q^{2} S R=e^{-i \hbar} S R$ imply that $f\left(e^{i \hbar} R\right) S=S f(R)$. In this section we give the precise meaning to the latter formula and find a class of functions $f$ for which it holds. In what follows we use functional calculus of selfadjoint operators. If $R$ is a selfadjoint operator and $f$ is a measurable function defined on $\operatorname{Sp} R$, then by definition

$$
f(R)=\int f(\lambda) d E_{R}(\lambda),
$$

where $d E_{R}(\lambda)$ is the spectral measure associated with $R$. If ker $R=\{0\}$, then $E_{R}(\{0\})=0$ and $f(\lambda)$ need not be defined at $\lambda=0$. Using the involution (1.4) 
we have

$$
f(R)^{*}=f^{*}\left(e^{i \hbar} R\right)
$$

for any $f \in \mathcal{H}$ and any selfadjoint operator $R$. We start with the following:

Proposition 3.1. Let $R$ and $S$ be selfadjoint operators acting on a Hilbert space $H$ such that $\operatorname{ker} R=\{0\}, R \multimap S$ and $g$ be a bounded function on $\Omega_{\hbar}$. Assume that $g \in \mathcal{H}$. Then $g(R) D(S)$ subset $D(S)$ and for any $x \in D(S)$ we have

$$
g\left(e^{i \hbar} R\right) S x=S g(R) x .
$$

Remark 3.1. If $R$ is strictly positive, then it is sufficient to have function $g$ defined on $\Omega_{\hbar}^{+}$only. Similarly if $R<0$ then $g$ may be restricted to $\Omega_{\hbar}^{-}$.

Proof. For $S=0$ the statement is trivial. Remembering that $\operatorname{sign} S$ commutes with $R$ we may assume that $S$ is strictly positive.

Let $\Sigma$ be the strip $\{\tau \in \mathbf{C}: 0<\Im \tau<1\}$ and $\mathcal{H}(\Sigma)$ be the space of all continuous functions on $\bar{\Sigma}$, holomorphic on $\Sigma$. Clearly $\mathcal{H}(\Sigma)$ endowed with the sup norm is a Banach space.

For any $\lambda \in \mathbf{R}$ and $\tau \in \Sigma$ we set: $\varphi_{\lambda}(\tau)=g\left(e^{\hbar \tau} \lambda\right)$. Then $\varphi_{\lambda} \in \mathcal{H}(\Sigma)$ and $\left\|\varphi_{\lambda}\right\| \leq C$, where $C=\sup \left\{|g(r)|: r \in \Omega_{\hbar}\right\}$. Therefore

$$
\int_{\mathbf{R}} \varphi_{\lambda} d \mu(\lambda) \in \mathcal{H}(\Sigma)
$$

for any (complex valued) finite measure $d \mu(\lambda)$ on $\mathbf{R}$.

Let $d E_{R}(\lambda)$ be the spectral measure of the selfadjoint operator $R, x, y \in D(S)$ and $d \mu(\lambda)=\left(y \mid d E_{R}(\lambda) S x\right)$. Then

$$
\int_{\mathbf{R}} \varphi_{\lambda}(\tau) d \mu(\lambda)=\left(y \mid g\left(e^{\hbar \tau} R\right) S x\right)
$$

and formula (3.3) shows that the function

$$
\Sigma \ni \tau \longrightarrow\left(y \mid g\left(e^{\hbar \tau} R\right) S x\right) \in \mathbf{C}
$$

is continuous and holomorphic on the interior of $\Sigma$.

Remembering that $x, y \in D(S)$ one can easily show that the mappings

$$
\begin{aligned}
& \bar{\Sigma} \ni \tau \longrightarrow S^{1+i \tau} x \in H, \\
& \bar{\Sigma} \ni \tau \longrightarrow S^{i \bar{\tau}} y \in H
\end{aligned}
$$

are continuous. The first one is holomorphic whereas the second one is antiholomorphic on $\Sigma$. Therefore the function

$$
\bar{\Sigma} \ni \tau \longrightarrow\left(S^{i \bar{\tau}} y \mid g(R) S^{1+i \tau} x\right) \in \mathbf{C}
$$

is continuous and holomorphic on $\Sigma$. 
Assume for the moment that $\tau$ is real. Using (2.3) we obtain

$$
\left(S^{i \bar{\tau}} y \mid g(R) S^{1+i \tau} x\right)=\left(y \mid S^{-i \tau} g(R) S^{1+i \tau} x\right)=\left(y \mid g\left(e^{\hbar \tau} R\right) S x\right) .
$$

By holomorphic continuation this equality holds for all $\tau \in \bar{\Sigma}$. In particular for $\tau=i$ we obtain

$$
(S y \mid g(R) x)=\left(y \mid g\left(e^{i \hbar} R\right) S x\right) .
$$

This relation holds for all $y \in D(S)$. Remembering that $S$ is selfadjoint we see that $g(R) x \in D(S)$ and $S g(R) x=g\left(e^{i \hbar} R\right) S x$.

Let $R \multimap S$, where $(R, S)$ is a pair of selfadjoint operators acting on the same Hilbert space $H$ such that $\operatorname{ker} R=\{0\}$. We shall use the function $\ell(\cdot)$ introduced by (1.2). Then for any $\lambda>0$ the operator $e^{-\lambda \ell(R)^{2}}$ is bounded and converges strongly to $I$, when $\lambda \rightarrow+0$. Therefore the domain

$$
D_{0}=\bigcup_{\lambda>0} e^{-\lambda \ell(R)^{2}} D(S),
$$

is dense in $H$. With this notation we have:

Proposition 3.2. Let $f \in \mathcal{H}$. Then

(0) $D_{0} \subset D(f(R))$

(1) $f(R) D_{0} \subset D_{0}$,

(2) $D_{0} \subset D(S)$,

(3) $S D_{0} \subset D\left(f\left(e^{i \hbar} R\right)\right)$.

Proof. Let $\lambda>0$. One can easily verify that the function $g(r)=e^{-\lambda \ell(r)^{2}}$ satisfies the assumptions of Proposition 3.1. Therefore $e^{-\lambda \ell(R)^{2}} D(S) \subset D(S)$ and Statement (2) follows.

Now, let $f \in \mathcal{H}$ and $\lambda>0$. Then the function $g(r)=f(r) e^{-\lambda \ell(r)^{2}}$ is bounded. Therefore $e^{-\lambda \ell(R)^{2}} H \subset D(f(R))$ and Statement (0) follows. One can easily verify that the function $g(r)$ considered in this paragraph satisfies the assumptions of Proposition 3.1. Therefore

$$
\begin{gathered}
f(R) e^{-\lambda \ell(R)^{2}} D(S) \subset D(S), \\
f(R) e^{-2 \lambda \ell(R)^{2}} D(S) \subset e^{-\lambda \ell(R)^{2}} D(S) \subset D_{0}
\end{gathered}
$$

and Statement (1) follows.

Let $x \in D_{0}$. Then

$$
x=e^{-\lambda \ell(R)^{2}} x^{\prime},
$$

where $x^{\prime} \in D(S)$ and $\lambda>0$. Formula (3.2) shows that

$$
e^{-\lambda \ell\left(e^{i \hbar} R\right)^{2}} S x^{\prime}=S x .
$$

If $f \in \mathcal{H}$ then the function $f(r) e^{-\lambda \ell(r)^{2}}$ is bounded, $f\left(e^{i \hbar} R\right) e^{-\lambda \ell\left(e^{i \hbar} R\right)^{2}} \in B(H)$ and $S x=e^{-\lambda \ell\left(e^{i \hbar} R\right)^{2}} S x^{\prime} \in D\left(f\left(e^{i \hbar} R\right)\right)$. Statement $(3)$ is proved. 
Let $(R, S)$ be a pair of selfadjoint operators acting on a Hilbert space $H$ such that $R \multimap S$ and $\operatorname{ker} R=\{0\}$, and let $f \in \mathcal{H}$. Proposition 3.2 shows that the compositions $S \circ f(R)$ and $f\left(e^{i \hbar} R\right) \circ S$ are densely defined (their domains contain $\left.D_{0}\right)$. Taking into account $(3.1)$ we see that the adjoints $(S \circ f(R))^{*} \supset f^{*}\left(e^{i \hbar} R\right) \circ S$ and $\left(f\left(e^{i \hbar} R\right) \circ S\right)^{*} \supset S \circ f^{*}(R)$ are densely defined. Therefore $S \circ f(R)$ and $f\left(e^{i \hbar} R\right) \circ S$ are closeable operators. In what follows their closures will be denoted by $S f(R)$ and $f\left(e^{i \hbar} R\right) S$.

Theorem 3.1. Let $(R, S)$ be a pair of selfadjoint operators acting on a Hilbert space $H$ such that $R \multimap S$ and ker $R=\{0\}$, and $D_{0}$ be the domain introduced by (3.4). Then for any $f \in \mathcal{H}$ we have:

(0) $D_{0}$ is a core for $f\left(e^{i \hbar} R\right) S$,

(1) $\left(f\left(e^{i \hbar} R\right) S\right)^{*}=S f^{*}(R)$,

(2) $f\left(e^{i \hbar} R\right) S \subset S f(R)$,

(3) If moreover $f^{-1} \in \mathcal{H}$, then $f\left(e^{i \hbar} R\right) S=S f(R)$,

(4) If $f^{*}=f$, then $f\left(e^{i \hbar} R\right) S$ is symmetric,

(5) If $f^{-1} \in \mathcal{H}$ and $f^{*}=f$, then $f\left(e^{i \hbar} R\right) S$ is selfadjoint and $R \multimap f\left(e^{i \hbar} R\right) S$.

If $R>0$ ( $R<0$ respectively $)$, then one may replace $\mathcal{H}$ by $\mathcal{H}^{+}\left(\mathcal{H}^{-}\right.$respectively $)$.

Proof Ad (1) and (0). We already know that $\left(f\left(e^{i \hbar} R\right) S\right)^{*} \supset S f^{*}(R)$. Therefore it is sufficient to prove the converse inclusion. Let $y \in D\left(\left(f\left(e^{i \hbar} R\right) S\right)^{*}\right)$ and $z=$ $\left(f\left(e^{i \hbar} R\right) S\right)^{*} x$. Then

$$
\left(y \mid f\left(e^{i \hbar} R\right) S x\right)=(z \mid x)
$$

for any $x \in D\left(f\left(e^{i \hbar} R\right) S\right.$ ). In particular (cf. Statements (3) and (4) of Proposition 3.2) this relation holds for all $x \in D_{0}$. Therefore

$$
\left(y \mid f\left(e^{i \hbar} R\right) S e^{-\lambda \ell(R)^{2}} x^{\prime}\right)=\left(z \mid e^{-\lambda \ell(R)^{2}} x^{\prime}\right)
$$

for any $\lambda>0$ and $x^{\prime} \in D(S)$. Using (3.2) we get

$$
\left(y \mid f\left(e^{i \hbar} R\right) e^{-\lambda \ell\left(e^{i \hbar} R\right)^{2}} S x^{\prime}\right)=\left(z \mid e^{-\lambda \ell(R)^{2}} x^{\prime}\right)
$$

and

$$
\left(f^{*}(R) e^{-\lambda \ell^{*}(R)^{2}} y \mid S x^{\prime}\right)=\left(e^{-\lambda \ell(R)^{2}} z \mid x^{\prime}\right) .
$$

This relation holds for all $x^{\prime} \in D(S)$. Remembering that $S$ is selfadjoint we obtain $f^{*}(R) e^{-\lambda \ell^{*}(R)^{2}} y \in D(S), e^{-\lambda \ell^{*}(R)^{2}} y \in D\left(S f^{*}(R)\right)$ and

$$
S f^{*}(R) e^{-\lambda \ell^{*}(R)^{2}} y=e^{-\lambda \ell(R)^{2}} z
$$

This relation holds for any $\lambda>0 . S f^{*}(R)$ is a closed operator. Setting $\lambda \rightarrow+0$, we obtain: $y \in D\left(S f^{*}(R)\right)$ and $S f^{*}(R) y=z$. This way we showed that $\left(f\left(e^{i \hbar} R\right) S\right)^{*} \subset$ $S f^{*}(R)$ and Statement (1) follows.

The reader should notice that using the relation (3.6) we restricted the range of variable $x$ to the set $D_{0}$. It shows that $f\left(e^{i \hbar} R\right) S$ and its restriction to $D_{0}$ have the same adjoint. Therefore they have the same closure. In other words, $D_{0}$ is a core for $f\left(e^{i \hbar} R\right) S$. 
Ad (2). Let $x \in D_{0}$. Then $x=e^{-\lambda \ell(R)^{2}} x^{\prime}$, where $x^{\prime} \in D(S)$ and $\lambda>0$. Formula (3.2) shows that $S f(R) x=f\left(e^{i \hbar} R\right) e^{-\lambda \ell\left(e^{i \hbar} R\right)^{2}} S x^{\prime}$. In particular for $f=1$ we get $S x=e^{-\lambda \ell\left(e^{i \hbar} R\right)^{2}} S x^{\prime}$. Comparing the two formulae we obtain

$$
f\left(e^{i \hbar} R\right) S x=S f(R) x .
$$

This formula holds for all $x \in D_{0}$. Remembering that $D_{0}$ is a core for $f\left(e^{i \hbar} R\right) S$ we obtain $f\left(e^{i \hbar} R\right) S \subset S f(R)$.

Ad (3). It is sufficient to show that $D_{0}$ is a core for $S f(R)$. Let $x \in D(S \circ f(R))$. Then $x \in D(f(R))$ and $y=f(R) x \in D(S)$. Therefore $f(R) e^{-\lambda \ell(R)^{2}} x=e^{-\lambda \ell(R)^{2}} y \in$ $D_{0}$ for any $\lambda>0$. In this point we assume that $f^{-1} \in \mathcal{H}$. By Statement (1) of Proposition 3.2, $f(R)^{-1} D_{0} \subset D_{0}$ and

$$
e^{-\lambda \ell(R)^{2}} x \in D_{0} \text {. }
$$

Using (3.2) we obtain

$$
S f(R) e^{-\lambda \ell(R)^{2}} x=e^{-\lambda \ell\left(e^{i \hbar} R\right)^{2}} S f(R) x .
$$

The reader should notice that $e^{-\lambda \ell(R)^{2}} x\left(e^{-\lambda \ell\left(e^{i \hbar} R\right)^{2}} S f(R) x\right.$ respectively) converges to $x$ (to $S f(R) x$ respectively) when $\lambda \rightarrow+0$. Taking into account (3.7) we see that $D_{0}$ is a core for $S f(R)$.

Ad (4) and (5). Inserting $f^{*}=f$ in Statement (1) and using Statement (2) we get $f\left(e^{i \hbar} R\right) S \subset\left(f\left(e^{i \hbar} R\right) S\right)^{*}$. It means that $f\left(e^{i \hbar} R\right) S$ is symmetric. If in addition $f^{-1} \in \mathcal{H}$ then (cf. Statement (3)) instead of inclusion we have equality and $f\left(e^{i \hbar} R\right) S$ is selfadjoint. To prove the Zakrzewski relation $R \multimap f\left(e^{i \hbar} R\right) S$ it is sufficient to notice that $\operatorname{sign} R$ and $|R|^{i \tau}$ commute with $f\left(e^{i \hbar} R\right)$.

Let $f \in \mathcal{H}$. One can easily verify that $f^{*}=f$ if and only if $f\left(e^{i \hbar / 2} r\right)$ is real for any $r \in \mathbf{R}$. Assume that this is the case and that $f^{-1} \in \mathcal{H}$. Then $f$ has no zeroes on $\Omega_{\hbar}$ and $\operatorname{sign} f\left(e^{i \hbar / 2} r\right.$ ) (for real $r$ ) depends only on $\operatorname{sign} r$. If $f\left( \pm e^{i \hbar / 2}\right)>0$, then $f=g^{2}$, where $g=\sqrt{f}$ and the branch of the square root is chosen in such a way that $g\left( \pm e^{i \hbar / 2}\right)>0$. Clearly $g \in \mathcal{H}$ and $g=g^{*}$. Then $g\left(e^{i \hbar} R\right)^{*}=g(R)$, $g\left(e^{i \hbar} R\right) S \subset S g(R)$ and for any $x \in D_{0}$ we have

$$
\left(x \mid f\left(e^{i \hbar} R\right) S x\right)=\left(x \mid g\left(e^{i \hbar} R\right)^{2} S x\right)=(g(R) x \mid S g(R) x) .
$$

Remembering that $D_{0}$ is a core for $f\left(e^{i \hbar} R\right) S$ and that the operators $R$ and $S$ respect the direct sum decomposition $H=H(S<0) \oplus H(S=0) \oplus H(S>0)$ we see that $\operatorname{sign} f\left(e^{i \hbar} R\right) S$ coincides with $\operatorname{sign} S$. One can easily modify the above considerations to include all four possible combinations of signs of $f\left( \pm e^{i \hbar / 2}\right)$. This way we obtain:

Theorem 3.2. Let $(R, S)$ be a pair of selfadjoint operators acting on a Hilbert space $H$ such that $R \multimap S$ and $\operatorname{ker} R=\{0\}$ and $f$ be a function on $\Omega_{\hbar}$ such that $f$, $f^{-1} \in \mathcal{H}$ and $f=f^{*}$. Then

$$
\operatorname{sign} f\left(e^{i \hbar} R\right) S=\left(\operatorname{sign} f\left(e^{i \hbar / 2} R\right)\right)(\operatorname{sign} S) .
$$

If $R>0(R<0$ respectively $)$, then one may replace $\Omega_{\hbar}$ and $\mathcal{H}$ by $\Omega_{\hbar}^{+}$and $\mathcal{H}^{+}\left(\Omega_{\hbar}^{-}\right.$ and $\mathcal{H}^{-}$respectively). 
Example 3.1. Let $f(r)=e^{-i \hbar / 2} r$. Then $f, f^{-1} \in \mathcal{H}$ and $f^{*}=f$. Therefore $e^{i \hbar / 2} R S$ is selfadjoint, sign $e^{i \hbar / 2} R S=(\operatorname{sign} R)(\operatorname{sign} S)$ and $R \multimap e^{i \hbar / 2} R S$. One can easily verify that $e^{i \hbar / 2} R S \multimap S$. The reader should notice that we need not assume that $\operatorname{ker} R=\{0\}$. Indeed the assertions we made are trivial if $R=0$ (this is the case not covered by Theorems 3.1 and 3.2).

Assume now that $R$ and $S$ are strictly positive. So is $e^{i \hbar / 2} R S$. We claim that

$$
\begin{aligned}
\left.\left(e^{i \hbar / 2} R S\right)^{i k}\right) & =e^{-\frac{i \hbar}{2} k^{2}} R^{i k} S^{i k} \\
& =e^{\frac{i \hbar}{2} k^{2}} S^{i k} R^{i k} .
\end{aligned}
$$

Indeed, using (2.2) one can easily show that the second equality holds and that $\left(e^{-\frac{i \hbar}{2} k^{2}} R^{i k} S^{i k}\right)_{k \in \mathbf{R}}$ is a one parameter group of unitaries. Therefore there exists a strictly positive operator $T$ such that

$$
T^{i k}=e^{-\frac{i \hbar}{2} k^{2}} R^{i k} S^{i k}
$$

for all $k \in \mathbf{R}$. Performing analytical continuation and setting $k=-i$ we obtain $T=e^{i \hbar / 2} R S$ and (3.8) follows.

\section{Properties of $\left(I+e^{i \hbar / 2} R\right) S$}

Throughout this section $R$ and $S$ are selfadjoint operators acting on a Hilbert space $H$ such that $R \multimap S$. We shall consider the operator $Q=\left(e^{i \hbar / 2} R+I\right) S$. By definition (cf. previous section) $Q$ is the closure of the composition $Q_{0}=\left(e^{i \hbar / 2} R+\right.$ $I) \circ S$. We shall prove that $Q_{0}$ is closed.

Let $\left(x_{n}\right)_{n \in \mathbf{N}}$ be a sequence of elements of $D\left(Q_{0}\right)$ converging to a vector $x_{\infty} \in H$ such that $\left(Q_{0} x_{n}\right)_{n \in \mathbf{N}}$ converges to a vector $y \in H$. Then $x_{n} \in D(S), S x_{n} \in D(R)$ and $\left(e^{i \hbar / 2} R+I\right) S x_{n} \rightarrow y$. The selfadjointness of $R$ implies that $-e^{-i \hbar / 2} \notin \operatorname{Sp} R$. Therefore the operator $e^{i \hbar / 2} R+I$ has a bounded inverse and $S x_{n} \rightarrow\left(e^{i \hbar / 2} R+I\right)^{-1} y$. Remembering that $S$ is closed we see that $x_{\infty} \in D(S)$ and $S x_{\infty}=\left(e^{i \hbar / 2} R+I\right)^{-1} y$. Therefore $S x_{\infty} \in D(R), x_{\infty} \in D\left(Q_{0}\right)$ and $Q_{0} x_{\infty}=\left(e^{i \hbar / 2} R+I\right) S x_{\infty}=y$. It shows that $Q_{0}$ is closed, $Q=Q_{0}$ and

$$
D(Q)=\{x \in D(S): S x \in D(R)\}=D\left(e^{i \hbar / 2} R S\right) \cap D(S) .
$$

Therefore

$$
Q=e^{i \hbar / 2} R S+S .
$$

Proposition 4.1. The operator $Q=e^{i \hbar / 2} R S+S$ is symmetric. The adjoint $Q^{*}=S\left(I+e^{-i \hbar / 2} R\right)$. If $R \geq 0$ then $Q$ is selfadjoint.

Proof. If $R=0$, then $\left(I+e^{i \hbar / 2} R\right) S=S$ is clearly selfadjoint. Therefore we may assume that $\operatorname{ker} R=\{0\}$. Let $f(r)=1+e^{-i \hbar / 2} r$. Then $f \in \mathcal{H}$ and $f^{*}=f$. Therefore (cf. Statement (4) of Theorem 3.1) the operator $\left(I+e^{i \hbar / 2} R\right) S$ is symmetric. The formula for $Q^{*}$ follows from Statement (2) of the same theorem. The function $f^{-1}$ has the only pole at the point $-e^{i \hbar / 2} \in \Omega_{\hbar}^{-}$. It means that $f^{-1} \in \mathcal{H}^{+}$. Therefore, by Statement (5) Theorem 3.1, the operator $\left(I+e^{i \hbar / 2} R\right) S$ is selfadjoint provided $R>0$. 
We shall see later that for $R<0$ the operator $\left(I+e^{i \hbar / 2} R\right) S$ is not selfadjoint. If in addition $S$ is of definite $\operatorname{sign}(S>0$ or $S<0)$ then $\left(I+e^{i \hbar / 2} R\right) S$ is maximal symmetric but not selfadjoint. In these cases $Q$ is unitarily equivalent to the differential operator: $-i \hbar \frac{d}{d s}$ on the real half-line $\left(\mathbf{R}_{+}=\{s \in \mathbf{R}: s>0\}\right.$ if $S>0$ and $\mathbf{R}_{-}=\{s \in \mathbf{R}: s<0\}$ if $\left.S<0\right)$.

By definition the differential operator $-i \hbar \frac{d}{d s}$ acts on the Hilbert space $L^{2}\left(\mathbf{R}_{ \pm}, K\right)$, where $K$ is a Hilbert space. Elements of $L^{2}\left(\mathbf{R}_{ \pm}, K\right)$ are square integrable (with respect to the Lebesque measure) functions $\mathbf{R}_{ \pm} \ni s \rightarrow x(s) \in K$. The domain $D\left(-i \hbar \frac{d}{d s}\right)$ consists of all continuous functions $x \in L^{2}\left(\mathbf{R}_{ \pm}, K\right)$ such that the derivative (in the sense of the distribution theory) $\frac{d x(s)}{d s}$ belongs to $L^{2}\left(\mathbf{R}_{ \pm}, K\right)$ and $\lim _{s \rightarrow 0} x(s)=0$. It is well known [1] that $-i \hbar \frac{d}{d s}$ is maximal symmetric but not selfadjoint. We shall also use the multiplication operator $\hat{s}:(\hat{s} x)(s)=s x(s)$. A function $x \in D(\hat{s})$ iff the function $s x(s)$ is square integrable.

Proposition 4.2. Assume that $R<0$ and $S>0$. Then there exists a unitary operator $U: H \rightarrow L^{2}\left(\mathbf{R}_{+}, K\right)$ such that

$$
\begin{gathered}
U \log (-R) U^{*}=-\frac{i \hbar}{2} I+i \hbar \frac{d}{d s} \hat{s}, \\
U\left(I+e^{i \hbar / 2} R\right) S U^{*}=-i \hbar \frac{d}{d s} .
\end{gathered}
$$

In the above formulae, $K$ is a Hilbert space of dimension equal to the multiplicity of $(R, S)$. The similar Statement holds for $S<0$. In this case we have to replace $\mathbf{R}_{+}$by $\mathbf{R}_{-}$.

Proof. For any $r \in \Omega_{\hbar}^{-}$we set $f(r)=\frac{-\left(1+e^{-i \hbar / 2} r\right)}{\log \left(-e^{-i \hbar / 2} r\right)}$, where the branch of the logarithm is chosen in such a way that the denominator vanishes for $r=-e^{i \hbar / 2}$. Then $f, f^{-1} \in \mathcal{H}$ and $f^{*}=f$. Moreover

$$
f\left(-e^{i \hbar / 2}\right)=\lim _{r \rightarrow-e^{i \hbar / 2}} \frac{-\left(1+e^{-i \hbar / 2} r\right)}{\log \left(-e^{-i \hbar / 2} r\right)}=1 .
$$

Therefore the operator $S^{\prime}=\frac{-\left(I+e^{i \hbar / 2} R\right)}{\log \left(-e^{i \hbar / 2} R\right)} S$ is selfadjoint, $\operatorname{sign} S^{\prime}=\operatorname{sign} S$ and $R \multimap S^{\prime}$.

We shall assume that $S>0$ (The case $S<0$ may be treated in the same way). Then $S^{\prime}>0$. Combining Theorem 2.1 with (2.7) we see that there exists a unitary $U: H \rightarrow L^{2}\left(\mathbf{R}_{+}, K\right)$ such that

$$
U \log (-R) U^{*}=-\frac{i \hbar}{2} I+i \hbar \frac{d}{d s} \hat{s}
$$

and

$$
U S^{\prime} U^{*}=\hat{s}^{-1} .
$$

By the first formula $U \log \left(-e^{i \hbar / 2} R\right) U^{*}=i \hbar \frac{d}{d s} \hat{s}$. Combining this result with the second formula we obtain

$$
U\left(I+e^{i \hbar / 2} R\right) S U^{*}=-U \log \left(-e^{i \hbar / 2} R\right) S^{\prime} U^{*}=-i \hbar \frac{d}{d s}
$$


We have to verify that the domain of this operator coincides with the one described above. According to Statement (0) of Theorem 3.1, the set

$$
U D_{0}=U \bigcup_{\lambda>0} e^{-\lambda \ell(R)^{2}} D\left(S^{\prime}\right)=\bigcup_{\lambda>0} \exp \left\{\lambda\left(\frac{I}{2}+\hat{s} \frac{d}{d s}\right)^{2}\right\} D\left(\hat{s}^{-1}\right)
$$

is a core for (4.2). Let $x \in L^{2}\left(\mathbf{R}_{+}, K\right)$ and $x(\lambda, s)$ be the value of the function

$$
x_{\lambda}=\exp \left\{\lambda\left(\frac{I}{2}+\hat{s} \frac{d}{d s}\right)^{2}\right\} x
$$

at the point $s$. Then $x(\lambda, s)$ satisfies the heat equation

$$
\frac{\partial}{\partial \lambda} x(\lambda, s)=\left(\frac{1}{2}+s \frac{\partial}{\partial s}\right)^{2} x(\lambda, s) .
$$

By the general theory of parabolic equations, for $\lambda>0$ and $s \neq 0$ the function $x(\lambda, s)$ is smooth in both variables. If $x \in D\left(\hat{s}^{-1}\right)$, then in a certain sense $x(s)$ approaches 0 when $s$ tends to 0 . In that case $\lim _{s \rightarrow 0} x(\lambda, s)=0$. It shows that elements $x \in D_{0}$ are continuous, smooth for $s \neq 0$ functions satisfying the boundary condition $x(0)=0$. Remembering that $D_{0}$ is a core for (4.2) one can show that the domain of (4.2) consists of all functions $x$ that are square integrable continuous functions on $\mathbf{R}_{+}$such that $x(0)=0$ with the derivative (in the sense of the distribution theory) being square integrable.

We end this section with a few formulae describing the domains of considered operators. According to Statement (1) of Theorem $3.1\left(\left(I+e^{i \hbar / 2} R\right) S\right)^{*}=$ $S\left(I+e^{-i \hbar / 2} R\right)$. By definition $S\left(I+e^{-i \hbar / 2} R\right)$ is the closure of the composition $S \circ\left(I+e^{-i \hbar / 2} R\right)$. One should not expect that $D\left(S\left(I+e^{-i \hbar / 2} R\right)\right)$ coincides with $D\left(S \circ\left(I+e^{-i \hbar / 2} R\right)\right)$. However we have

$$
D(R) \cap D\left(S\left(I+e^{-i \hbar / 2} R\right)\right)=\left\{x \in D(R):\left(I+e^{-i \hbar / 2} R\right) x \in D(S)\right\} .
$$

Only the inclusion LHS $\subset$ RHS needs a proof (the converse is obvious). Let $x \in D(R)$ and $x \in D\left(S\left(I+e^{-i \hbar / 2} R\right)\right)$. According to Statements (2) and (3) of Proposition $3.2 D_{0} \subset D\left(\left(I+e^{i \hbar / 2} R\right) \circ S\right)$. For any element $y \in D_{0}$ we have

$$
\left(y \mid S\left(I+e^{-i \hbar / 2} R\right) x\right)=\left(\left(I+e^{i \hbar / 2} R\right) S y \mid x\right)=\left(S y \mid\left(I+e^{-i \hbar / 2} R\right) x\right) .
$$

By Statement (0) of Theorem 3.1, $D_{0}$ is a core for $S$. Therefore $\left(I+e^{-i \hbar / 2} R\right) x \in$ $D(S)$ and (4.4) holds.

If $(R, S)$ is a pair of selfadjoint operators satisfying the Zakrzewski relation with a given $\hbar$, then $(S, R)$ satisfies the same relation with $\hbar$ replaced by $-\hbar$. Applying this remark to (4.4) we obtain

$$
D(S) \cap D\left(R\left(I+e^{i \hbar / 2} S\right)\right)=\left\{x \in D(S):\left(I+e^{i \hbar / 2} S\right) x \in D(R)\right\} .
$$

Let $x \in D(S) \cap D\left(S\left(I+e^{-i \hbar / 2} R\right)\right)$. Then for any $y \in D_{0}$ we have

$$
\left(y \mid S\left(I+e^{-i \hbar / 2} R\right) x\right)=\left(\left(I+e^{i \hbar / 2} R\right) S y \mid x\right)=(S y \mid x)+\left(e^{i \hbar / 2} R S y \mid x\right)
$$


and

$$
\left(e^{i \hbar / 2} R S y \mid x\right)=\left(y \mid S\left(I+e^{-i \hbar / 2} R\right) x\right)-(S y \mid x)=\left(y \mid S\left(I+e^{-i \hbar / 2} R\right) x-S x\right) .
$$

By Statement (0) of Theorem 3.1, $D_{0}$ is a core for $e^{i \hbar / 2} R S$. Therefore $x \in$ $D\left(e^{i \hbar / 2} R S\right)$ and

$$
D(S) \cap D\left(S\left(I+e^{-i \hbar / 2} R\right)\right) \subset D(S) \cap D\left(e^{i \hbar / 2} R S\right)=D\left(S+e^{i \hbar / 2} R S\right) .
$$

The converse inclusion is trivial: $S\left(I+e^{-i \hbar / 2} R\right)=\left(S+e^{i \hbar / 2} R S\right)^{*}$ is an extension of the symmetric operator $S+e^{i \hbar / 2} R S$.

\section{Selfadjoint Extensions}

Let $R$ and $S$ be selfadjoint operators on a Hilbert space $H$ satisfying the Zakrzewski relation $R \multimap S$ and $Q=\left(e^{i \hbar / 2} R+I\right) S$. According to (4.1), $Q$ is a sum of two selfadjoint operators. Therefore $Q$ is symmetric. We already know that in general $Q$ is not selfadjoint. In what follows, the selfadjoint extensions of $Q$ will play a very essential role. We start with the following general considerations.

Let $H$ be a Hilbert space, $Q$ be a symmetric operator acting on $H$ and $\rho$ be a selfadjoint operator acting on $H$ such that $\rho^{2}$ is a projection. We say that $\rho$ is a reflection operator for $Q$ if $\rho$ anticommutes with $Q$ and $Q$ restricted to $H(\rho=0)$ is selfadjoint. By definition $\rho$ anticommutes with $Q$ iff $\rho Q \subset-Q \rho$. If this is the case then $Q$ respects the direct sum decomposition

$$
H=H(\rho=0) \oplus H\left(\rho^{2}=1\right)
$$

and the restriction of $Q$ to $H(\rho=0)$ is well defined.

Proposition 5.1. Let $Q$ be a symmetric operator acting on a Hilbert space $H$ and $\rho$ be a reflection operator for $Q$. Then there exists a unique selfadjoint extension $Q_{\rho}$ of $Q$ such that

$$
(\rho-I) D\left(Q_{\rho}\right) \subset D(Q)
$$

The operator $Q_{\rho}$ coincides with the restriction of $Q^{*}$ to the domain

$$
\begin{aligned}
D_{\rho} & =D(Q)+D\left(Q^{*}\right) \cap H(\rho=1) \\
& =\left\{x \in D\left(Q^{*}\right):(\rho-I) x \in D(Q)\right\} .
\end{aligned}
$$

Proof. We know that $\operatorname{Sp} \rho=\{-1,0,1\}$. Therefore

$$
H=H(\rho=-1) \oplus H(\rho=0) \oplus H(\rho=1)
$$

and elements of $x \in H$ may be written in the column form

$$
x=\left(\begin{array}{c}
x_{-} \\
x_{0} \\
x_{+}
\end{array}\right),
$$


where $x_{-} \in H(\rho=-1), x_{0} \in H(\rho=0)$ and $x_{+} \in H(\rho=1)$. Consequently the operator $\rho$ should be written as matrix

$$
\rho=\left(\begin{array}{ccc}
-I & 0 & 0 \\
0 & 0 & 0 \\
0 & 0 & I
\end{array}\right)
$$

Using the anticommutativity of $Q$ with (5.5), one can easily show that operator $Q$ is of the form

$$
Q=\left(\begin{array}{ccc}
0 & 0 & Q_{+} \\
0 & Q_{0} & 0 \\
Q_{-} & 0 & 0
\end{array}\right)
$$

where

$$
\begin{gathered}
Q_{+}: H(\rho=1) \rightarrow H(\rho=-1), \\
Q_{0}: H(\rho=0) \rightarrow H(\rho=0), \\
Q_{-}: H(\rho=-1) \rightarrow H(\rho=1)
\end{gathered}
$$

are closed densely defined operators. The domain

$$
D(Q)=\left\{\begin{array}{cc}
x \in H: & x \text { is of the form (5.4), where } \\
x_{-} \in D\left(Q_{-}\right), x_{0} \in D\left(Q_{0}\right), x_{+} \in D\left(Q_{+}\right)
\end{array}\right\} .
$$

Since $\rho$ is a reflection operator for $Q$, the restriction of $Q$ to $H(\rho=0)$ is selfadjoint: $Q_{0}=Q_{0}^{*}$. The adjoint operator

$$
Q^{*}=\left(\begin{array}{ccc}
0 & 0 & Q_{-}^{*} \\
0 & Q_{0} & 0 \\
Q_{+}^{*} & 0 & 0
\end{array}\right)
$$

has the domain

$$
D\left(Q^{*}\right)=\left\{\begin{array}{cc}
x \in H: & x \text { is of the form (5.4), where } \\
x_{-} \in D\left(Q_{+}^{*}\right), x_{0} \in D\left(Q_{0}\right), x_{+} \in D\left(Q_{-}^{*}\right)
\end{array}\right\} .
$$

Operator $Q$ is symmetric. Therefore

$$
Q_{+} \subset Q_{-}^{*}
$$

Let

$$
Q_{\rho}=\left(\begin{array}{ccc}
0 & 0 & Q_{-}^{*} \\
0 & Q_{0} & 0 \\
Q_{-} & 0 & 0
\end{array}\right)
$$

Then $Q_{\rho}$ is selfadjoint. Relation (5.8) shows that $Q \subset Q_{\rho} \subset Q^{*}$. The domain

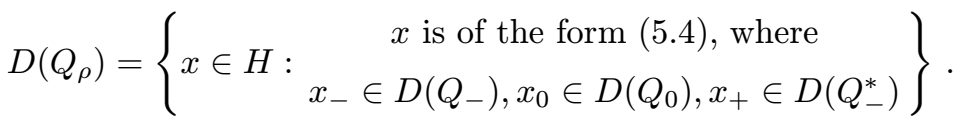


Remembering that $(\rho-I)$ kills the last component of (5.4) and using (5.6) we see that (5.2) holds. Comparing (5.9) with (5.6) and (5.7) we see that $D\left(Q_{\rho}\right)$ coincides with (5.3). It shows that $Q_{\rho}$ is the restriction of $Q^{*}$ to $D_{\rho}$. This way the existence of the extension $Q \subset Q_{\rho}$ satisfying the condition (5.2) is established. We shall prove the uniqueness.

Let $Q_{\rho}^{\prime}$ be a selfadjoint extension of $Q$. Then $Q_{\rho}^{\prime} \subset Q^{*}$ and $D\left(Q_{\rho}^{\prime}\right) \subset D\left(Q^{*}\right)$. If $(\rho-I) D\left(Q_{\rho}^{\prime}\right) \subset D(Q)$, then using the second expression of (5.3) we see that $D\left(Q_{\rho}^{\prime}\right) \subset D_{\rho}$ and $Q_{\rho}^{\prime} \subset Q_{\rho}$. Passing to the adjoint operators we get the converse inclusion $Q_{\rho}^{\prime} \supset Q_{\rho}$. It shows that $Q_{\rho}^{\prime}=Q_{\rho}$.

Only for a very limited class of symmetric operators $Q$, the procedure described in the above proposition gives all selfadjoint extensions of $Q$. Among them we have symmetric operators of the form (4.1).

Proposition 5.2. Let $(R, S)$ be a pair of selfadjoint operators acting on a Hilbert space $H$ such that $R \multimap S$ and $Q=e^{i \hbar / 2} R S+S$. Assume that $\rho$ is a selfadjoint operator such that

$$
\left.\begin{array}{c}
\rho^{2}=\chi(R<0), \\
\rho \text { commutes with } R \text { and } \\
\rho \text { anticommutes with } S
\end{array}\right\} \text {. }
$$

Then $\rho$ is a reflection operator for $Q$. Any selfadjoint extension of $Q$ is related (via Proposition 5.1) to a reflection operator $\rho$ satisfying conditions (5.10). If $\operatorname{ker} S=$ $\{0\}$, then the operator $\rho$ is determined uniquely by the extension.

Proof. By the first relation of (5.10), $\rho^{2}$ is a projection and $H(\rho=0)=H(R \geq 0)$. We already know (cf. Proposition 4.1) that $Q$ is selfadjoint for $R \geq 0$. Therefore $Q$ restricted to $H(\rho=0)$ is selfadjoint. Due to (5.10) $\rho$ anticommutes with $Q$. It shows that $\rho$ is a reflection operator for $Q$.

We have to show that any selfadjoint extension of $Q$ is of the form $Q_{\rho}$. To this end it is sufficient to consider the case $R<0$. Then the operator $\rho$ is a unitary involution: $\rho^{*} \rho=\rho \rho^{*}=\rho^{2}=I$. We shall also assume that $\operatorname{ker} S=\{0\}$ (for $S=0$ the statement is trivial, in this case $Q=0)$. Then $H=H(S<0) \oplus H(S>0)$. Taking into account Proposition 4.2 we may assume that $H(S<0)=L^{2}\left(\mathbf{R}_{-}, K_{-}\right)$ and $H(S>0)=L^{2}\left(\mathbf{R}_{+}, K_{+}\right)$, where $K_{-}$and $K_{+}$are Hilbert spaces of dimensions equal to the multiplicity of negative and positive part of the spectrum of $S$. With this identification

$$
\begin{aligned}
\log (-R) & =-\frac{i \hbar}{2} I+i \hbar \frac{d}{d s} \hat{s}, \\
Q & =-i \hbar \frac{d}{d s} .
\end{aligned}
$$

These operators act on the Hilbert space $H=L^{2}\left(\mathbf{R}_{-}, K_{-}\right) \oplus L^{2}\left(\mathbf{R}_{+}, K_{+}\right)$. Elements $x \in H$ are square integrable functions on $\mathbf{R}$ with values $x(s) \in K_{-}$for $s<0$ and $x(s) \in K_{+}$for $s>0$. Elements $x \in D(Q)$ are square integrable continuous functions on $\mathbf{R}$ such that $x(0)=0$ with the derivative (in the sense of the distribution theory) 
being square integrable. The adjoint operator $Q^{*}$ is given by the same formula (5.12). Its domain consists of all square integrable functions $x(s)$ on $\mathbf{R}$ continuous for $s \neq 0$ with the derivative being square integrable. For any $x \in D\left(Q^{*}\right)$ there exist limits

$$
x( \pm 0)=\lim _{s \rightarrow \pm 0} x(s)
$$

Clearly $x(-0) \in K_{-}$and $x(+0) \in K_{+}$. An element $x \in D\left(Q^{*}\right)$ belongs to $D(Q)$ if and only if $x( \pm 0)=0$.

Any selfadjoint extension of a symmetric operator $Q$ is a restriction of its adjoint $Q^{*}$. Using the general theory of selfadjoint extensions of differential operators [1] one can easily show that there is a one to one correspondence between the set of all selfadjoint extensions of (5.12) and the set of unitary operators acting from $K_{-}$ onto $K_{+}$. Let $u: K_{-} \rightarrow K_{+}$be a unitary operator. Then restricting $Q^{*}$ to the domain

$$
D_{u}=\left\{x \in D\left(Q^{*}\right): x(+0)=u x(-0)\right\}
$$

we obtain the corresponding selfadjoint extension $Q_{u}$ of $Q$.

Let $\rho$ be a selfadjoint operator satisfying (5.10). Then $\rho$ is a unitary involution commuting with $R$ and anticommuting with $Q$. Using (5.11) and (5.12) one can easily show that $\rho$ anticommutes with $\hat{s}$. Consequently there exists two families of unitaries $u_{s} \in B\left(K_{-}, K_{+}\right)$and $v_{s} \in B\left(K_{+}, K_{-}\right)$(where $s \in \mathbf{R}_{+}$) such that

$$
(\rho x)(s)= \begin{cases}u_{s} x(-s) & \text { for } s>0 \\ v_{-s} x(-s) & \text { for } s<0\end{cases}
$$

for any $x \in H$. Relation $\rho^{2}=\chi(R<0)=I$ shows that $v_{s}=u_{s}^{*}$. Using once more the anticommutativity of $\rho$ and (5.12) one can easily prove that $u_{s}=u$ does not depend on $s$. This way we showed that the operator $\rho$ is of the form

$$
(\rho x)(s)= \begin{cases}u x(-s) & \text { for } s>0 \\ u^{*} x(-s) & \text { for } s<0\end{cases}
$$

In this formula $u \in B\left(K_{-}, K_{+}\right)$is a fixed (independent of $x$ ) unitary operator and $x \in H$. Conversely it is not difficult to verify that any operator $\rho$ of the form (5.14) is a selfadjoint operator satisfying (5.10).

We have to show that (5.13) coincides with (5.3). Let $x \in D\left(Q^{*}\right) \cap H(\rho=1)$. Then $\rho x=x$ and $u x(-s)=u(s)$ for any $s>0$. Letting $s \rightarrow 0$ we get $u x(-0)=$ $x(+0)$. Therefore $x \in D_{u}$ and $D_{\rho} \subset D_{u}$. Conversely assume that $x \in D_{u}$. Then $x \in D\left(Q^{*}\right)$ and $x(+0)=u x(-0)$. The latter is equivalent to $(x-\rho x)( \pm 0)=0$ and to $x-\rho x \in D(Q)$. Clearly $x+\rho x \in D\left(Q^{*}\right) \cap H(\rho=1)$. Now the decomposition $2 x=(x-\rho x)+(x+\rho x)$ shows that $x \in D_{\rho}$ and $D_{u} \subset D_{\rho}$.

According to (5.10) $R$ and $\rho$ do commute and the joint spectrum

$$
\operatorname{Sp}(R, \rho)=\mathbf{R}_{-} \times\{-1,1\} \cup \mathbf{R}_{+} \times\{0\}
$$


is contained in the closure of the domain $\Delta_{F}$ of $F_{\hbar}$ (cf. Theorem 1.1). In what follows, we shall extend $F_{\hbar}$ to the closure $\overline{\Delta_{F}}$ by setting

$$
F_{\hbar}(0, \varrho)=1
$$

for $\varrho=0, \pm 1$. With this extension $F_{\hbar}$ remains a continuous function (cf. (1.8)). The main result of this section is contained in the following theorem.

Theorem 5.1. Let $R$ and $S$ be selfadjoint operators acting on a Hilbert space $H$ such that $R \multimap S, \rho$ be a selfadjoint operator satisfying (5.10) and $Q_{\rho}$ be the selfadjoint extension of $Q=\left(e^{i \hbar / 2} R+I\right) S$ corresponding to the reflection operator $\rho$. Then

$$
Q_{\rho}=F_{\hbar}(R, \rho)^{*} S F_{\hbar}(R, \rho),
$$

where $F_{\hbar}: \Delta_{F} \rightarrow \mathbf{C}$ is a function satisfying conditions (1)-(4) of Theorem 1.1.

Proof. At first we notice that the statement is trivial for $S=0$ so we may assume that $\operatorname{ker} S=\{0\}$. Moreover all the operators involved in the theorem commute with $\operatorname{sign} R$. Therefore they respect the direct sum decomposition

$$
H=H(R<0) \oplus H(R=0) \oplus H(R>0)
$$

and it is sufficient to consider separately three cases: $R<0, R=0$ and $R>0$. If $R=0$, then $Q=S$ and the statement is trivial.

Assume now that $R>0$. In this case (cf. Proposition 4.1) $Q$ is selfadjoint, $\rho=0$ and we have to show that

$$
Q=F_{\hbar}(R, 0)^{*} S F_{\hbar}(R, 0) .
$$

For any $r \in \Omega_{\hbar}^{+}$we set: $f(r)=F_{\hbar}(r, 0)$. Then (cf. Condition (2) of Theorem 1.1) $f, f^{-1} \in \mathcal{H}^{+}$. According to (1.5) and (1.6), $f(R)=F_{\hbar}(R, 0)$ is a unitary operator and $f\left(e^{i \hbar} R\right)=\left(e^{i \hbar / 2} R+I\right) F_{\hbar}(R, 0)$. Statement (3) of Theorem 3.1 shows that

$$
\left(e^{i \hbar / 2} R+I\right) F_{\hbar}(R, 0) S=S F_{\hbar}(R, 0)
$$

and (5.16) follows.

Assume now that $R<0$. In this case $\operatorname{Sp} \rho=\{-1,1\}$. For any $r \in \Omega_{\hbar}^{-}$we set: $f_{ \pm}(r)=F_{\hbar}(r, \pm 1)$. Then (cf. Condition (3) of Theorem 1.1) $f_{+}, f_{-}, f_{-}^{-1} \in$ $\mathcal{H}^{-}$. According to (1.5) and (1.6), $f_{ \pm}(R)=F_{\hbar}(R, \pm 1)$ are unitary operators and $f_{ \pm}\left(e^{i \hbar} R\right)=\left(e^{i \hbar / 2} R+I\right) F_{\hbar}(R, \mp 1)$. Statements (2) and (3) of Theorem 3.1 show that

$$
\begin{aligned}
& \left(e^{i \hbar / 2} R+I\right) F_{\hbar}(R,-1) S \subset S F_{\hbar}(R, 1), \\
& \left(e^{i \hbar / 2} R+I\right) F_{\hbar}(R, 1) S=S F_{\hbar}(R,-1) .
\end{aligned}
$$

Consequently

$$
\begin{aligned}
& Q \subset F_{\hbar}(R,-1)^{*} S F_{\hbar}(R, 1), \\
& Q=F_{\hbar}(R, 1)^{*} S F_{\hbar}(R,-1) .
\end{aligned}
$$


Remembering that $\rho$ anticommutes with $Q$, one can easily show that

$$
D(Q)=D(Q) \cap H(\rho=1)+D(Q) \cap H(\rho=-1) .
$$

Let $x \in D(Q) \cap H(\rho= \pm 1)$. Then

$$
F_{\hbar}(R, \rho) x=F_{\hbar}(R, \pm 1) x \in D(S) \cap H(\rho= \pm 1) .
$$

Remembering that $S$ anticommutes with $\rho$ we have

$$
S F_{\hbar}(R, \rho) x=S F_{\hbar}(R, \pm 1) x \in H(\rho=\mp 1)
$$

and taking into account (5.17) and (5.18) we obtain: $x \in D(Q)$ and

$$
F_{\hbar}(R, \rho)^{*} S F_{\hbar}(R, \rho) x=F_{\hbar}(R, \mp 1)^{*} S F_{\hbar}(R, \pm 1) x=Q x .
$$

Formula (5.19) shows now that $F_{\hbar}(R, \rho)^{*} S F_{\hbar}(R, \rho)$ is a selfadjoint extension of $Q$. To end the proof it is sufficient to notice that in the relation (5.18) we have strict equality. It shows that

$$
(\rho-1) D\left(F_{\hbar}(R, \rho)^{*} S F_{\hbar}(R, \rho)\right) \subset D(Q)
$$

Therefore (cf. condition (5.2)) $F_{\hbar}(R, \rho) * S F_{\hbar}(R, \rho)$ is the extension related to the reflection operator $\rho$.

Now we are able to show that Conditions (1)-(5) of Theorem 1.1 determine the function $F_{\hbar}$ uniquely. If $F_{\hbar}$ and $F_{\hbar}^{\prime}$ are functions satisfying these conditions, then formula (5.15) holds for both functions. Consequently $F_{\hbar}^{\prime}(R, \rho) F_{\hbar}(R, \rho)^{-1}$ commutes with $S$ and with $|S|^{i t}(t \in \mathbf{R})$ :

$$
F_{\hbar}^{\prime}(R, \rho) F_{\hbar}(R, \rho)^{-1}=|S|^{-i t} F_{\hbar}^{\prime}(R, \rho) F_{\hbar}(R, \rho)^{-1}|S|^{i t}
$$

According to (5.10), $S$ anticommutes with $\rho$. Therefore $|S|$ commutes with $\rho$ and $|S|^{-i t} \rho|S|^{i t}=\rho$. Taking into account (2.3) we obtain

$$
F_{\hbar}^{\prime}(R, \rho) F_{\hbar}(R, \rho)^{-1}=F_{\hbar}^{\prime}(\lambda R, \rho) F_{\hbar}(\lambda R, \rho)^{-1}
$$

where $\lambda=e^{\hbar t}$ is a positive number. Therefore

$$
F_{\hbar}^{\prime}(r, \varrho) F_{\hbar}(r, \varrho)^{-1}=F_{\hbar}^{\prime}(\lambda r, \varrho) F_{\hbar}(\lambda r, \varrho)^{-1}
$$

for any $(r, \varrho) \in \Delta_{F}$ such that $r \in \mathbf{R}$. Letting $\lambda \rightarrow+0$ and using (1.7) (for $F_{\hbar}$ and $\left.F_{\hbar}^{\prime}\right)$ we obtain $F_{\hbar}^{\prime}(r, \varrho) F_{\hbar}(r, \varrho)^{-1}=1$ and $F_{\hbar}^{\prime}(r, \varrho)=F_{\hbar}(r, \varrho)$. By analytical continuation this equality holds for all $(r, \varrho) \in \Delta_{F}$.

We would like to rewrite the results of Secs. 4 and 5 in a slightly different setting.

Theorem 5.2. Let $R$ and $S$ be selfadjoint operators satisfying the relation $R \multimap S$. Then:

(1) Operator $R+S$ is a closed symmetric operator.

(2) $R+S$ is selfadjoint if and only if $e^{i \hbar / 2} R S \geq 0$. 
(3) If $R$ and $S$ are of opposite sign (e.g. $R>0$ and $S<0)$ then $R+S$ is maximal symmetric but not selfadjoint.

(4) Any selfadjoint extension of $R+S$ is of the form $[R+S]_{\tau}$, where $\tau$ is a selfadjoint operator such that $\tau$ anticommutes with $R$ and $S$ and $\tau^{2}=\chi\left(e^{i \hbar / 2} R S<0\right)$. The reflection operator $\tau$ is defined uniquely by the extension.

(5) If $\operatorname{ker} S=\{0\}$, then

$$
[R+S]_{\tau}=F_{\hbar}\left(e^{i \hbar / 2} S^{-1} R, \tau\right)^{*} S F_{\hbar}\left(e^{i \hbar / 2} S^{-1} R, \tau\right) .
$$

(6) If $\operatorname{ker} R=\{0\}$, then

$$
[R+S]_{\tau}=F_{\hbar}\left(e^{i \hbar / 2} S R^{-1}, \tau\right) R F_{\hbar}\left(e^{i \hbar / 2} S R^{-1}, \tau\right)^{*}
$$

Proof. For $S=0$ the all six statements are trivial. Therefore we may assume that $\operatorname{ker} S=\{0\}$. In this case $\operatorname{sign} e^{i \hbar / 2} S^{-1} R=(\operatorname{sign} S)(\operatorname{sign} R)=\operatorname{sign} e^{i \hbar / 2} R S$, $\chi\left(e^{i \hbar / 2} S^{-1} R \geq 0\right)=\chi\left(e^{i \hbar / 2} R S \geq 0\right)$ and $e^{i \hbar / 2} S^{-1} R \geq 0$ if and only if $e^{i \hbar / 2} R S \geq 0$.

Let $R^{\prime}=e^{i \hbar / 2} S^{-1} R$. Then $R^{\prime}$ is selfadjoint, $R^{\prime} \multimap S$ and $R+S=\left(e^{i \hbar / 2} R^{\prime}+\right.$ $I) S$. Statement (1) and the "if" part of Statement (2) follow immediately from Proposition 4.1. Statement (3) follows from Proposition 4.2. Statement (4) follows from Proposition 5.2 and Statement (5) from (5.15). If $(R, S)$ is a pair of selfadjoint operators satisfying the Zakrzewski relation with a given $\hbar$, then $(S, R)$ satisfies the same relation with $\hbar$ replaced by $-\hbar$. Applying this remark to Statement (5) and using (1.16) we obtain Statement (6).

We shall prove the "only if" part of Statement (2). The reader should notice that multiplying a reflection operator by -1 we obtain a new reflection operator. If $R+S$ is selfadjoint then by Statement (4) the set of reflection operators contains precisely one element $\tau$. In this case $\tau=-\tau, \tau=0$ and $\chi\left(e^{i \hbar / 2} S^{-1} R<0\right)=\tau^{2}=0$. It shows that $e^{i \hbar / 2} S^{-1} R \geq 0$.

\section{Exponential Equality}

The following theorem shows that the name Quantum exponential function given to $F_{\hbar}$ is justified (cf. formula $(6.5)$ ).

Theorem 6.1. Let $(R, S)$ be a pair of selfadjoint operators acting on a Hilbert space $H$ such that $R \multimap S$ and $\operatorname{ker} S=\{0\}$, and $\rho, \sigma \in B(H)$. Assume that $\rho$ and $\sigma$ are selfadjoint and

$$
\left.\begin{array}{c}
\rho^{2}=\chi(R<0), \\
\rho \text { commutes with } R \text { and } \\
\rho \text { anticommutes with } S,
\end{array}\right\}
$$


We set

$$
\begin{aligned}
& T=e^{i \hbar / 2} S^{-1} R, \\
& \tau=\alpha \rho \sigma+\bar{\alpha} \sigma \rho,
\end{aligned}
$$

where $\alpha$ is the constant introduced by (1.10). Then

(1) $T$ is selfadjoint, $\operatorname{sign} T=(\operatorname{sign} R)(\operatorname{sign} S), T \multimap R$ and $T \multimap S$

(2) $\tau$ is selfadjoint and

$$
\left.\begin{array}{c}
\tau^{2}=\chi(T<0), \\
\tau \text { commutes with } T \text { and } \\
\tau \text { anticommutes with } R \text { and } S
\end{array}\right\}
$$

(3) $\tau$ is a reflection operator for $R+S$ and

$$
\begin{aligned}
F_{\hbar}(R, \rho) F_{\hbar}(S, \sigma) & =F_{\hbar}(T, \tau)^{*} F_{\hbar}(S, \sigma) F_{\hbar}(T, \tau) \\
& =F_{\hbar}\left([R+S]_{\tau}, \widetilde{\sigma}\right)
\end{aligned}
$$

where $[R+S]_{\tau}$ is the selfadjoint extension of $R+S$ corresponding to the reflection operator $\tau$ and $\widetilde{\sigma}=F_{\hbar}(T, \tau)^{*} \sigma F_{\hbar}(T, \tau)$.

Remark 6.1. Let $\hat{p}$ and $\hat{q}$ be selfadjoint quantum mechanical position and momentum operators introduced in Sec. 2. Then operators: $R=e^{i \hbar / 2} e^{\hat{p}} e^{\hat{q}}=e^{\hat{p}+\hat{q}}, S=e^{\hat{q}}$, $\rho=\sigma=0$ satisfy the assumptions of Theorem 6.1 . In this case $T=e^{i \hbar / 2} S^{-1} R=e^{\hat{p}}$, $\tau=0$ and the relation (6.5) shows that

$$
V_{\frac{2 \pi}{\hbar}}(\hat{p}) V_{\frac{2 \pi}{\hbar}}(\hat{p}+\hat{q}) V_{\frac{2 \pi}{\hbar}}(\hat{q})=V_{\frac{2 \pi}{\hbar}}(\hat{q}) V_{\frac{2 \pi}{\hbar}}(\hat{p}) .
$$

This formula was presented (without proof) in the lecture of Kashaev [5].

Proof of Theorem 6.1. If $R=0$ then $\rho=0$ and $T=0$. In this case the Statements are trivial. Therefore we may assume that $\operatorname{ker} R=\{0\}$.

Ad (1). At first we notice that $S^{-1} \multimap R$. This fact follows immediately from Proposition 2.1. Using Example 3.1 at the end of Sec. 3 we see that $T$ is selfadjoint, $\operatorname{sign} T=(\operatorname{sign} R)(\operatorname{sign} S), T \multimap R$ and $S^{-1} \multimap T$. According to Proposition 2.1 the last relation shows that $T \multimap S$.

Ad (2). To understand better the formulae (6.1), (6.2) and (6.4) we shall use the following matrix notation. We know that the kernels of $R$ and $S$ are trivial and that signs of $R$ and $S$ do commute. Therefore setting

$$
\begin{aligned}
& H_{++}=H(R>0, S>0), \\
& H_{+-}=H(R>0, S<0), \\
& H_{-+}=H(R<0, S>0), \\
& H_{--}=H(R<0, S<0)
\end{aligned}
$$


we have

$$
H=H_{++} \oplus H_{+-} \oplus H_{-+} \oplus H_{--}
$$

and elements of $x \in H$ may be written in the column form

$$
x=\left(\begin{array}{c}
x_{++} \\
x_{+-} \\
x_{-+} \\
x_{--}
\end{array}\right)
$$

where $x_{i j} \in H_{i j}, i, j \in\{+,-\}$. Consequently the operators acting on $H$ will be represented by $4 \times 4$ matrices.

Using (6.1) one can easily show that $\rho$ kills $H_{++}$and $H_{+-}$and that $\rho: H_{--} \rightarrow$ $H_{-+}$and $\rho: H_{-+} \rightarrow H_{--}$are mutually inverse unitary maps. Similarly (6.2) shows that $\sigma$ kills $H_{++}$and $H_{-+}$and that $\sigma: H_{--} \rightarrow H_{+-}$and $\sigma: H_{+-} \rightarrow H_{--}$ are mutually inverse unitary maps. Using these unitaries we may identify the three Hilbert spaces: $H_{-+}$and $H_{+_{-}}$with $H_{--}$. In what follows $H_{-+}=H_{+-}=H_{--}$will be denoted by $H_{o}$. With this identification, the operators $\rho$ and $\sigma$ are represented by matrices:

$$
\rho=\left(\begin{array}{llll}
0 & 0 & 0 & 0 \\
0 & 0 & 0 & 0 \\
0 & 0 & 0 & I \\
0 & 0 & I & 0
\end{array}\right), \quad \sigma=\left(\begin{array}{llll}
0 & 0 & 0 & 0 \\
0 & 0 & 0 & I \\
0 & 0 & 0 & 0 \\
0 & I & 0 & 0
\end{array}\right)
$$

Performing elementary matrix computations we obtain

$$
\tau=\alpha \rho \sigma+\bar{\alpha} \sigma \rho=\left(\begin{array}{cccc}
0 & 0 & 0 & 0 \\
0 & 0 & \bar{\alpha} & 0 \\
0 & \alpha & 0 & 0 \\
0 & 0 & 0 & 0
\end{array}\right) .
$$

Operators $R$ and $S$ do commute with $\operatorname{sign} R$ and $\operatorname{sign} S$. Therefore they are represented by diagonal matrices. Remembering that $\rho$ commutes with $R$ and $\sigma$ commutes with $S$ we obtain

$$
R=\left(\begin{array}{cccc}
R_{+} & 0 & 0 & 0 \\
0 & R_{o} & 0 & 0 \\
0 & 0 & -R_{o} & 0 \\
0 & 0 & 0 & -R_{o}
\end{array}\right), \quad S=\left(\begin{array}{cccc}
S_{+} & 0 & 0 & 0 \\
0 & -S_{o} & 0 & 0 \\
0 & 0 & S_{o} & 0 \\
0 & 0 & 0 & -S_{o}
\end{array}\right),
$$

where $R_{+}$and $S_{+}$are restrictions of $R$ and $S$ to $H_{++}$and $R_{0}$ and $S_{0}$ are restrictions of $-R$ and $-S$ to $H_{--}=H_{o}$. Clearly $R_{+}, S_{+}, R_{0}$ and $S_{0}$ are strictly positive selfadjoint operators, $R_{+} \multimap S_{+}$and $R_{0} \multimap S_{0}$. Multiplying the two matrices we get

$$
T=e^{i \hbar / 2} S^{-1} R=\left(\begin{array}{cccc}
T_{+} & 0 & 0 & 0 \\
0 & -T_{o} & 0 & 0 \\
0 & 0 & -T_{o} & 0 \\
0 & 0 & 0 & T_{o}
\end{array}\right)
$$


where $T_{+}=e^{i \hbar / 2} S_{+}^{-1} R_{+}$and $T_{o}=e^{i \hbar / 2} S_{o}^{-1} R_{o}$ are strictly positive selfadjoint operators.

Now, using the matrix forms of $\tau, T, R$ and $S$ derived above, one can easily check that $\tau$ is selfadjoint and that conditions (6.4) hold.

Ad (3). In the second part of this section we shall derive the following formula:

$$
\begin{aligned}
& F_{\hbar}(T, \tau)^{*} F_{\hbar}(S, \sigma)^{*} T^{-1} F_{\hbar}(S, \sigma) F_{\hbar}(T, \tau) \\
& \quad=F_{\hbar}(S, \sigma)^{*} F_{\hbar}(R, \rho)^{*} T^{-1} F_{\hbar}(R, \rho) F_{\hbar}(S, \sigma) .
\end{aligned}
$$

It means that the unitary operator $F_{\hbar}(S, \sigma) F_{\hbar}(T, \tau) F_{\hbar}(S, \sigma)^{*} F_{\hbar}(R, \rho)^{*}$ commutes with $T^{-1}$. Therefore it commutes with $|T|$ and $|T|^{i t}$ :

$$
\begin{aligned}
& F_{\hbar}(S, \sigma) F_{\hbar}(T, \tau) F_{\hbar}(S, \sigma)^{*} F_{\hbar}(R, \rho)^{*} \\
& \quad=|T|^{i t} F_{\hbar}(S, \sigma) F_{\hbar}(T, \tau) F_{\hbar}(S, \sigma)^{*} F_{\hbar}(R, \rho)^{*}|T|^{-i t}
\end{aligned}
$$

for any $t \in \mathbf{R}$.

We already know that $T$ anticommutes with $\rho$ and $\sigma$ and commutes with $\tau$. Therefore $|T|$ commutes with $\rho, \sigma$ and $\tau$ and

$$
|T|^{i t} \rho|T|^{-i t}=\rho, \quad|T|^{i t} \sigma|T|^{-i t}=\sigma \quad \text { and } \quad|T|^{i t} \tau|T|^{-i t}=\tau .
$$

Clearly

$$
|T|^{i t} T|T|^{-i t}=T .
$$

Moreover the relations $T \multimap R$ and $T \multimap S$ show that

$$
|T|^{i t} R|T|^{-i t}=\lambda R \quad \text { and } \quad|T|^{i t} S|T|^{-i t}=\lambda S,
$$

where $\lambda=e^{\hbar t} \in \mathbf{R}^{+}$. Inserting these data into (6.12) we get

$$
\begin{aligned}
& F_{\hbar}(S, \sigma) F_{\hbar}(T, \tau) F_{\hbar}(S, \sigma)^{*} F_{\hbar}(R, \rho)^{*} \\
& \quad=F_{\hbar}(\lambda S, \sigma) F_{\hbar}(T, \tau) F_{\hbar}(\lambda S, \sigma)^{*} F_{\hbar}(\lambda R, \rho)^{*} .
\end{aligned}
$$

Let $\lambda \rightarrow+0$. Using (1.7) one can easily show that $F_{\hbar}(\lambda S, \sigma), F_{\hbar}(\lambda R, \rho)$ and their adjoints converge strongly to $I$. Therefore

$$
F_{\hbar}(S, \sigma) F_{\hbar}(T, \tau) F_{\hbar}(S, \sigma)^{*} F_{\hbar}(R, \rho)^{*}=F_{\hbar}(T, \tau)
$$

and

$$
F_{\hbar}(R, \rho) F_{\hbar}(S, \sigma)=F_{\hbar}(T, \tau)^{*} F_{\hbar}(S, \sigma) F_{\hbar}(T, \tau) .
$$

To end the proof we notice, that due to Theorem 5.1,

$$
F_{\hbar}(T, \tau)^{*} F_{\hbar}(S, \sigma) F_{\hbar}(T, \tau)=\left[e^{i \hbar / 2} T S+S\right]_{\tau}=[R+S]_{\tau} .
$$

The remaining part of this section is devoted to the proof of the formula (6.11). We shall keep the notations introduced in Theorem 6.1. 
We already know that $T \multimap S$. Therefore $S \multimap T^{-1}$. Inserting $\left(S, T^{-1}\right)$ instead of $(R, S)$ in $(4.4),(4.5)$ and in (4.6) we obtain

$$
\begin{aligned}
& D(S) \cap D\left(T^{-1}\left(I+e^{-i \hbar / 2} S\right)\right) \\
& =\left\{x \in D(S):\left(I+e^{-i \hbar / 2} S\right) x \in D\left(T^{-1}\right)\right\}, \\
& D\left(S\left(I+e^{i \hbar / 2} T^{-1}\right)\right) \cap D\left(T^{-1}\right) \\
& =\left\{x \in D\left(T^{-1}\right):\left(I+e^{i \hbar / 2} T^{-1}\right) x \in D(S)\right\}, \\
& D\left(T^{-1}\right) \cap D\left(\left(e^{i \hbar / 2} S T^{-1}+T^{-1}\right)^{*}\right) \\
& \subset D\left(e^{i \hbar / 2} S T^{-1}+T^{-1}\right) \text {. }
\end{aligned}
$$

Operator $T^{-1}$ is selfadjoint. Therefore $-e^{-i \hbar / 2} \notin \mathrm{Sp} T^{-1}$ and $I+e^{i \hbar / 2} T^{-1}$ is a closed operator with bounded inverse. The latter statement holds for $e^{-i \hbar / 2} I+S$ and for the product $\left(e^{-i \hbar / 2} I+S\right)\left(I+e^{i \hbar / 2} T^{-1}\right)$. Clearly

$$
\left\{\left(e^{-i \hbar / 2} I+S\right)\left(I+e^{i \hbar / 2} T^{-1}\right)\right\}^{*}=\left(I+e^{-i \hbar / 2} T^{-1}\right)\left(e^{i \hbar / 2} I+S\right) .
$$

Dealing with equalities of unbounded operators one has to verify that the domains of the operators are the same. Obviously

$$
D\left(\left(I+e^{-i \hbar / 2} T^{-1}\right)\left(e^{i \hbar / 2} I+S\right)\right)=\left\{x \in D(S):\left(e^{i \hbar / 2} I+S\right) x \in D\left(T^{-1}\right)\right\} .
$$

By (6.13), the same set is the domain of $S+T^{-1}\left(I+e^{-i \hbar / 2} S\right)$. Therefore

$$
\begin{aligned}
\left(I+e^{-i \hbar / 2} T^{-1}\right)\left(e^{i \hbar / 2} I+S\right) & =e^{i \hbar / 2} I+S+T^{-1}\left(I+e^{-i \hbar / 2} S\right) \\
& =e^{i \hbar / 2} I+S+\left[\left(I+e^{i \hbar / 2} S\right) T^{-1}\right]^{*} \\
& =e^{i \hbar / 2} I+S+\left[e^{i \hbar / 2} S T^{-1}+T^{-1}\right]^{*}
\end{aligned}
$$

where in the second step we used Statement (1) of Theorem 3.1 (with $R, S$ replaced by $\left.S, T^{-1}\right)$. In the same way, using (6.14) one can show that

$$
\left(e^{-i \hbar / 2} I+S\right)\left(I+e^{i \hbar / 2} T^{-1}\right)=e^{-i \hbar / 2} I+\left[S+e^{i \hbar / 2} S T^{-1}\right]^{*}+T^{-1} .
$$

Inserting these data into (6.16) we get

$$
\left\{\left[S+e^{i \hbar / 2} S T^{-1}\right]^{*}+T^{-1}\right\}^{*}=S+\left[e^{i \hbar / 2} S T^{-1}+T^{-1}\right]^{*} .
$$

Using (6.4) and (6.2) one can easily show that $\tau$ is a reflection operator for $S+$ $e^{i \hbar / 2} S T^{-1}$ and $\sigma$ is a reflection operator for $e^{i \hbar / 2} S T^{-1}+T^{-1}$. Let $\left[S+e^{i \hbar / 2} S T^{-1}\right]_{\tau}$ and $\left[e^{i \hbar / 2} S T^{-1}+T^{-1}\right]_{\sigma}$ be the corresponding selfadjoint extensions. Then

$$
\begin{gathered}
{\left[S+e^{i \hbar / 2} S T^{-1}\right]_{\tau} \subset\left[S+e^{i \hbar / 2} S T^{-1}\right]^{*},} \\
{\left[e^{i \hbar / 2} S T^{-1}+T^{-1}\right]_{\sigma} \subset\left[e^{i \hbar / 2} S T^{-1}+T^{-1}\right]^{*} .}
\end{gathered}
$$


Inserting these data into (6.17) and remembering that passing to the adjoint operators reverses the inclusion relation we get

$$
\left\{\left[S+e^{i \hbar / 2} S T^{-1}\right]_{\tau}+T^{-1}\right\}^{*} \supset S+\left[e^{i \hbar / 2} S T^{-1}+T^{-1}\right]_{\sigma} .
$$

Inserting in Theorem $5.1 T, e^{i \hbar / 2} S T^{-1}$ and $\tau$ instead of $R, S$ and $\rho$ we obtain

$$
\left[S+e^{i \hbar / 2} S T^{-1}\right]_{\tau}=F_{\hbar}(T, \tau)^{*} e^{i \hbar / 2} S T^{-1} F_{\hbar}(T, \tau)
$$

and remembering that $T^{-1}$ commutes with $F_{\hbar}(T, \tau)$ we get

$$
\left[S+e^{i \hbar / 2} S T^{-1}\right]_{\tau}+T^{-1}=F_{\hbar}(T, \tau)^{*}\left[e^{i \hbar / 2} S T^{-1}+T^{-1}\right] F_{\hbar}(T, \tau) .
$$

Combining this formula with (6.18) we have

$$
F_{\hbar}(T, \tau)^{*}\left[e^{i \hbar / 2} S T^{-1}+T^{-1}\right]^{*} F_{\hbar}(T, \tau) \supset S+\left[e^{i \hbar / 2} S T^{-1}+T^{-1}\right]_{\sigma} .
$$

We shall prove that

$$
D\left(\left[e^{i \hbar / 2} S T^{-1}+T^{-1}\right]_{\sigma}\right) \supset F_{\hbar}(T, \tau) D\left(S+\left[e^{i \hbar / 2} S T^{-1}+T^{-1}\right]_{\sigma}\right) .
$$

Let $Q=e^{i \hbar / 2} S T^{-1}+T^{-1}$ and

$$
x \in D\left(S+\left[e^{i \hbar / 2} S T^{-1}+T^{-1}\right]_{\sigma}\right) .
$$

Then $x \in D\left(Q_{\sigma}\right)$. Using (5.2) we obtain $(\sigma-I) x \in D(Q)$ and $(\sigma-I) x \in D\left(T^{-1}\right)$. We shall use the following simple formula:

$$
(\sigma-I) F_{\hbar}(T, \tau) x=(\sigma-I)\left[F_{\hbar}(T, \tau)-I\right] x+(\sigma-I) x .
$$

The second term belongs to $D\left(T^{-1}\right)$. By $(1.8),\left[F_{\hbar}(T, \tau)-I\right] x \in D\left(T^{-1}\right)$. The reader should notice that $\sigma$ commutes with $\left|T^{-1}\right|$. Therefore $D\left(T^{-1}\right)=D\left(\left|T^{-1}\right|\right)$ is invariant with respect to the action of $\sigma,(\sigma-I)\left[F_{\hbar}(T, \tau)-I\right] x \in D\left(T^{-1}\right)$ and

$$
(\sigma-I) F_{\hbar}(T, \tau) x \in D\left(T^{-1}\right) .
$$

Comparing (6.21) with (6.19) we obtain $F_{\hbar}(T, \tau) x \in D\left(Q^{*}\right)$. It is also easy to notice that $\sigma$ is a reflection operator for $Q$. Therefore (cf. the proof of Proposition 5.1) $D\left(Q^{*}\right)$ is $\sigma$-invariant and $(\sigma-I) F_{\hbar}(T, \tau) x \in D\left(Q^{*}\right)$. Now using (6.22) and (6.15) we see that $(\sigma-I) F_{\hbar}(T, \tau) x \in D(Q)$. Remembering that $F_{\hbar}(T, \tau) x \in D\left(Q^{*}\right)$ and using (5.3) we obtain $F_{\hbar}(T, \tau) x \in D\left(Q_{\sigma}\right)$ and (6.20) follows.

Combining (6.19) with (6.20) we obtain

$$
F_{\hbar}(T, \tau)^{*}\left[e^{i \hbar / 2} S T^{-1}+T^{-1}\right]_{\sigma} F_{\hbar}(T, \tau) \supset S+\left[e^{i \hbar / 2} S T^{-1}+T^{-1}\right]_{\sigma} .
$$

According to Theorem 5.1, $\left[e^{i \hbar / 2} S T^{-1}+T^{-1}\right]_{\sigma}=F_{\hbar}(S, \sigma)^{*} T^{-1} F_{\hbar}(S, \sigma)$. Remembering that $F_{\hbar}(S, \sigma)$ commutes with $S$ we obtain

$$
F_{\hbar}(T, \tau)^{*} F_{\hbar}(S, \sigma)^{*} T^{-1} F_{\hbar}(S, \sigma) F_{\hbar}(T, \tau) \supset F_{\hbar}(S, \sigma)^{*}\left[S+T^{-1}\right] F_{\hbar}(S, \sigma) .
$$


The left-hand side of the above relation is selfadjoint. Therefore

$$
F_{\hbar}(T, \tau)^{*} F_{\hbar}(S, \sigma)^{*} T^{-1} F_{\hbar}(S, \sigma) F_{\hbar}(T, \tau)=F_{\hbar}(S, \sigma)^{*} \widetilde{Q} F_{\hbar}(S, \sigma),
$$

where $\widetilde{Q}$ is a selfadjoint extension of $S+T^{-1}$. Using $(6.3)$ we see that $S=e^{i \hbar / 2} R T^{-1}$ and $S+T^{-1}=e^{i \hbar / 2} R T^{-1}+T^{-1}$. We know that $T \multimap R$. Therefore $R \multimap T^{-1}$. Now Proposition 5.2 and Theorem 5.1 show that

$$
\widetilde{Q}=\left[S+T^{-1}\right]_{\rho^{\prime}}=F_{\hbar}\left(R, \rho^{\prime}\right)^{*} T^{-1} F_{\hbar}\left(R, \rho^{\prime}\right),
$$

where $\rho^{\prime}$ is a selfadjoint operator such that

$$
\left.\begin{array}{c}
\rho^{\prime 2}=\chi(R<0), \\
\text { commutes with } R \text { and } \\
\text { nticommutes with } T^{-1} .
\end{array}\right\}
$$

One can easily show that these conditions are equivalent to (6.1) (with $\rho$ replaced by $\left.\rho^{\prime}\right)$. Inserting the value of $\widetilde{Q}$ into $(6.24)$ we obtain

$$
\begin{aligned}
& F_{\hbar}(T, \tau)^{*} F_{\hbar}(S, \sigma)^{*} T^{-1} F_{\hbar}(S, \sigma) F_{\hbar}(T, \tau) \\
& \quad=F_{\hbar}(S, \sigma)^{*} F_{\hbar}\left(R, \rho^{\prime}\right)^{*} T^{-1} F_{\hbar}\left(R, \rho^{\prime}\right) F_{\hbar}(S, \sigma) .
\end{aligned}
$$

This formula almost coincides with (6.11). Instead of $\rho$ we have $\rho^{\prime}$. To prove (6.11) we have to show that $\rho^{\prime}=\rho$.

We shall use the matrix notation introduced in the proof of Statement (2) of Theorem 6.1. One can easily show that any selfadjoint operator $\rho^{\prime}$ satisfying (6.25) is of the form

$$
\rho^{\prime}=\left(\begin{array}{cccc}
0 & 0 & 0 & 0 \\
0 & 0 & 0 & 0 \\
0 & 0 & 0 & \rho_{o} \\
0 & 0 & \rho_{o}^{*} & 0
\end{array}\right),
$$

where $\rho_{o}$ is a unitary operator acting on $H_{o}$ commuting with $R_{o}$ and $T_{o}$. We have to show that $\rho_{o}=I$. Assume for the moment that this is not the case.

Lemma 6.1. Assume that $\rho_{o} \neq I$. Then there exists a vector $y \in H_{o}$ such that $y \in D\left(T_{o}^{-1}\right), y \in D\left(\left(S_{o}-e^{i \hbar / 2} S_{o} T_{o}^{-1}\right)^{*}\right)$ and $\left(\rho_{o}-I\right) y \notin D\left(S_{o}\right)$.

Proof. Remembering the relation expressing $T_{o}$ in terms of $R_{o}$ and $S_{o}$ one can easily check that $\rho_{o}$ commutes with $S_{o}$. Using Proposition 4.1 with $R$ and $S$ replaced by $S_{o}$ and $T_{o}^{-1}$ we obtain

$$
\left(S_{o}-e^{i \hbar / 2} S_{o} T_{o}^{-1}\right)^{*}=S_{o}\left(I-e^{-i \hbar / 2} T_{o}^{-1}\right) .
$$

Operators $S_{o}$ and $T_{o}^{-1}$ are positive selfadjoint and $S_{o} \multimap T_{o}^{-1}$. Combining Theorem 2.1 with (2.6) we may assume that $H_{o}=L^{2}(\mathbf{R}, K), S_{o}$ is the multiplication by $e^{t}(t$ is the variable running over $\mathbf{R})$ and $T_{o}^{-1}$ is the imaginary shift by $i \hbar$ :

$$
\begin{gathered}
\left(S_{o} y\right)(t)=e^{t} y(t), \\
\left(T_{o}^{-1} y\right)(t)=y(t+i \hbar) .
\end{gathered}
$$


Remembering that $\rho_{o}$ commutes with $S_{o}$ and $T_{o}$ we conclude that it is of the form

$$
\left(\rho_{o} y\right)(t)=\varrho y(t)
$$

where $\varrho$ is a unitary operator acting on $K . \rho_{o} \neq I$ implies $\varrho \neq I$.

Let $y_{0}$ be an element of $K$ such that $(\varrho-I) y_{0} \neq 0$ and

$$
y(t)=\frac{y_{0}}{\cosh \left(\frac{t}{2}\right)} .
$$

The function $y$ is square integrable: $y \in L^{2}(\mathbf{R}, K)$. Noticing that the function $\frac{e^{t}(\varrho-I) y_{0}}{\cosh (t / 2)}$ is not square integrable we conclude that $(\rho-I) y_{0} \notin D\left(S_{o}\right)$. On the other hand the functions $y(t+i \hbar)$ and

$$
\begin{aligned}
e^{t}\left(y(t)-e^{-i \hbar / 2} y(t+i \hbar)\right)(t) & =e^{t}\left(\frac{1}{\cosh \left(\frac{t}{2}\right)}-\frac{e^{-i \hbar / 2}}{\cosh \left(\frac{t+i \hbar}{2}\right)}\right) y_{0} \\
& =\frac{\left(e^{-i \hbar / 2}-1\right) e^{\frac{3 t}{2}}}{\left(e^{\tau}+1\right)\left(e^{\tau}+e^{-i \hbar}\right)} y_{0}
\end{aligned}
$$

are square integrable. Therefore $y \in D\left(T_{o}^{-1}\right),\left(I-e^{-i \hbar / 2} T_{o}^{-1}\right) y \in D\left(S_{o}\right)$ and

$$
y \in D\left(\left(S_{o}-e^{i \hbar / 2} S_{o} T_{o}^{-1}\right)^{*}\right)
$$

We continue our proof of (6.11). Let $y \in H_{o}$ be the vector introduced in Lemma 6.1 and

$$
x=\left(\begin{array}{c}
0 \\
y \\
\alpha y \\
0
\end{array}\right) \in H .
$$

Then using (6.8), (6.10) and (6.9) one can easily verify that $\tau x=x, x \in D\left(T^{-1}\right)$ and $x \in D\left(\left(S+e^{i \hbar / 2} S T^{-1}\right)^{*}\right)$. Therefore (cf. formula (5.3) of Proposition 5.1)

$$
x \in D\left(\left[S+e^{i \hbar / 2} S T^{-1}\right]_{\tau}+T^{-1}\right) .
$$

The left-hand side of (6.24) coincides with that of (6.23). Therefore

$$
F_{\hbar}(T, \tau)^{*}\left[e^{i \hbar / 2} S T^{-1}+T^{-1}\right] F_{\hbar}(T, \tau) \subset F_{\hbar}(S, \sigma)^{*}\left[S+T^{-1}\right]_{\rho^{\prime}} F_{\hbar}(S, \sigma)
$$

and by Theorem 5.1,

$$
\left[S+e^{i \hbar / 2} S T^{-1}\right]_{\tau}+T^{-1} \subset F_{\hbar}(S, \sigma)^{*}\left[S+T^{-1}\right]_{\rho^{\prime}} F_{\hbar}(S, \sigma)
$$

Relation (6.29) shows now that $F_{\hbar}(S, \sigma) x \in D\left(\left[S+T^{-1}\right]_{\rho^{\prime}}\right)$. By virtue of (5.2), $\left(\rho^{\prime}-I\right) F_{\hbar}(S, \sigma) x \in D\left(S+T^{-1}\right)$ and

$$
\left(\rho^{\prime}-I\right) F_{\hbar}(S, \sigma) x \in D(S)
$$


Using (6.9), (6.7) and taking into account formula (1.17) we obtain

$$
F_{\hbar}(S, \sigma)=\left(\begin{array}{cccc}
U & 0 & 0 & 0 \\
0 & X & 0 & Y \\
0 & 0 & Z & 0 \\
0 & Y & 0 & X
\end{array}\right),
$$

where

$$
\begin{aligned}
& X=V_{\frac{2 \pi}{\hbar}}\left(\log \left(S_{o}\right)-i \pi I\right), \quad U=F_{\hbar}\left(S_{+}, 0\right) \\
& Y=i S_{o}^{\frac{\pi}{\hbar}} X=F_{\hbar}\left(-S_{o}, 1\right)-X, \quad Z=F_{\hbar}\left(S_{o}, 0\right) .
\end{aligned}
$$

Combining this formula with (6.27) and (6.28) we obtain

$$
\left(\rho^{\prime}-I\right) F_{\hbar}(S, \sigma) x=\left(\begin{array}{c}
0 \\
-X y \\
\rho_{o} Y y-\alpha Z y \\
\alpha \rho_{o} Z y-Y y
\end{array}\right) .
$$

This vector must belong to $D(S)$. In particular its second and fourth component belong to $D\left(S_{o}\right)$. Therefore $-X y+\alpha \rho_{o} Z y-Y y \in D\left(S_{o}\right)$ and

$$
\alpha \rho_{o} F_{\hbar}\left(S_{o}, 0\right) y-F_{\hbar}\left(-S_{o}, 1\right) y \in D\left(S_{o}\right) .
$$

Until this point in our proof, $\alpha$ could have been any complex number of modulus 1. Now we shall use the fact that this constant coincides with (1.10). By (1.11), $\alpha F_{\hbar}\left(S_{o}, 0\right) y-F_{\hbar}\left(-S_{o}, 1\right) y \in D\left(S_{o}\right)$ for any $y \in H_{o}$. Therefore $\left(\rho_{o}-I\right) F_{\hbar}\left(S_{o}, 0\right) y$ belongs to $D\left(S_{o}\right)$ and $\left(\rho_{o}-I\right) y \in D\left(S_{o}\right)\left(F_{\hbar}\left(S_{o}, 0\right)\right.$ is a unitary commuting with $\rho_{o}$ and $S_{o}$ ), which contradicts Lemma 6.1. It means that the assumption $\rho_{o} \neq I$ was wrong. It shows that $\rho_{o}=I, \rho^{\prime}=\rho$ and (6.26) coincides with (6.11). This ends the proof of (6.11).

\section{Exponential Equation}

In this section we shall prove that the function $F_{\hbar}$ is essentially the only function satisfying relation (6.5). Let

$$
\Delta_{\text {real }}=\{(r, \varrho) \in \Delta: r \in \mathbf{R}\} .
$$

Theorem 7.1. Let $R, S, \rho, \sigma, \tau$ and $\widetilde{\sigma}$ be operators considered in Sec. 6 (satisfying the assumptions of Theorem 6.1) and $f$ be a measurable complex valued function on $\Delta_{\text {real }}$ such that $|f(r, \varrho)|=1$ for any $(r, \varrho) \in \Delta_{\text {real }}$. Assume that $(-1) \in \operatorname{Sp} R$. Then the following two conditions are equivalent:

(1) Exponential equation:

$$
f(R, \rho) f(S, \sigma)=f\left([R+S]_{\tau}, \widetilde{\sigma}\right) .
$$


(2) There exists real non-negative $\mu$ and $s= \pm 1$ such that

$$
f(r, \varrho)=F_{\hbar}(\mu r, s \varrho)
$$

for almost ${ }^{\mathrm{a}}$ all $(r, \varrho) \in \Delta_{\text {real }}$.

Proof. At first we shall explain the role of the assumption saying that $(-1) \in \operatorname{Sp} R$. It shows that in the decomposition (6.6) one of the last two subspaces is not trivial. Therefore the space $H_{o}$ considered in the previous section is not trivial and the spectral measure of $S_{o}$ is equivalent to the Lebesgue measure on $\mathbf{R}_{+}$.

One can easily verify that the operators $R^{\prime}=\mu R, S^{\prime}=\mu S, \rho^{\prime}=s \rho$ and $\sigma^{\prime}=s \sigma$ satisfy the assumptions of Theorem 6.1. This substitution leaves $\tau$ unchanged: $\tau^{\prime}=\tau$ and changes the sign of $\widetilde{\sigma}: \widetilde{\sigma}^{\prime}=-\widetilde{\sigma}$. Inserting these data into (6.5) we see that the function (7.2) satisfies Eq. (7.1). This way we showed that (7.2) implies (7.1).

We shall prove the converse. By Theorem 5.1, relation (7.1) may be written in the following form (cf. 6.5)):

$$
f(R, \rho) f(S, \sigma)=F_{\hbar}(T, \tau)^{*} f(S, \sigma) F_{\hbar}(T, \tau) .
$$

We know that $|S|$ commutes with $\rho, \sigma, \tau$ and that $R \multimap S$ and $T \multimap S$. Therefore the unitary transformation $|S|^{i t \tau}$ leaves $\rho, \sigma, \tau$ and $S$ invariant and scales $R$ and $T$ by positive factor $e^{\hbar t}$. Replacing $e^{\hbar t}$ by $\lambda$

$$
f(\lambda R, \rho) f(S, \sigma)=F_{\hbar}(\lambda T, \tau)^{*} f(S, \sigma) F_{\hbar}(\lambda T, \tau)
$$

for any $\lambda>0$. After simple computation we get

$$
\frac{f(\lambda R, \rho)-I}{\lambda} f(S, \sigma)=F_{\hbar}(\lambda T, \tau)^{*} f(S, \sigma) \frac{F_{\hbar}(\lambda T, \tau)-I}{\lambda}+\frac{F_{\hbar}(\lambda T, \tau)^{*}-I}{\lambda} f(S, \sigma) .
$$

Using the asymptotic formula (1.8) one can easily show that for any $x \in D(T)$ we have

$$
\lim _{\lambda \rightarrow 0} \frac{F_{\hbar}(\lambda T, \tau)-I}{\lambda} x=\frac{1}{2 i \sin \frac{\hbar}{2}} T x .
$$

Therefore for any $x, y \in D(T)$ there exists limit:

$$
\lim _{\lambda \rightarrow 0}\left(x\left|\frac{f(\lambda R, \rho)-I}{\lambda}\right| f(S, \sigma) y\right)=\frac{1}{2 i \sin \frac{\hbar}{2}}\{(x \mid f(S, \sigma) T y)-(T x \mid f(S, \sigma) y)\} .
$$

We shall use the following

Lemma 7.1. Let $f$ be a bounded measurable function on $\mathbf{R}_{+}, R$ be a positive selfadjoint operator acting on a Hilbert space $H$ and $\Xi$ be the set of all pairs $(x, z)$, where $x \in D(R)$ and $z \in H$, such that there exists the limit:

$$
\Phi(x, z)=\lim _{\lambda \rightarrow+0}\left(x\left|\frac{f(\lambda R)-I}{\lambda}\right| z\right) .
$$

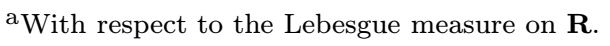


Assume that there exists a pair $(y, u) \in \Xi$ such that $(R y \mid u) \neq 0$. Then there exists a constant $\mu_{f}$ such that

$$
\Phi(x, z)=\mu_{f}(R x \mid z)
$$

for all $(x, z) \in \Xi$. The constant $\mu_{f}$ depends only on $f$; it is independent of $H$ and $R$.

Proof. Let $R=\int_{0}^{\infty} r d E(r)$ be the spectral decomposition of $R$ and $\varphi(r)=\frac{f(r)-1}{r}$ for any positive $r$. Then the formula (7.5) takes the following form:

$$
\Phi(x, z)=\lim _{\lambda \rightarrow+0} \int_{0}^{\infty} \varphi(\lambda r) d \mu_{x z}(r),
$$

where $d \mu_{x z}(r)=r(x|d E(r)| z)=(R x|d E(r)| z)$ is a finite measure.

Let $(x, z) \in \Xi$ and $(y, u) \in \Xi$. Remembering that $f$ is bounded we obtain $\left|\varphi\left(\lambda r r^{\prime}\right)\right|<\frac{\text { const }}{\lambda r r^{\prime}}$. On the other hand $\frac{1}{r r^{\prime}} d \mu_{x z}(r) d \mu_{y u}\left(r^{\prime}\right)=(x|d E(r)| z)(y|d E(r)| u)$ is a finite measure. Therefore the function $\varphi\left(\lambda r r^{\prime}\right)$ is integrable with respect to $d \mu_{x z}(r) d \mu_{y u}\left(r^{\prime}\right)$ and by Fubini's theorem:

$$
\int_{0}^{\infty}\left\{\int_{0}^{\infty} \varphi\left(\lambda r r^{\prime}\right) d \mu_{x z}(r)\right\} d \mu_{y u}\left(r^{\prime}\right)=\int_{0}^{\infty}\left\{\int_{0}^{\infty} \varphi\left(\lambda r r^{\prime}\right) d \mu_{y u}\left(r^{\prime}\right)\right\} d \mu_{x z}(r) .
$$

Let $\lambda \rightarrow 0$. Using the existence of the limit (7.7) one can easily show that the expression in braces on the left-hand side is bounded with the bound independent of $\lambda$ and $r^{\prime}$. By the Lebesque dominated convergence theorem the operation $\lim _{\lambda \rightarrow 0}$ commutes with the first integration. Using (7.7) we see that the left-hand side of (7.8) converges to $\Phi(x, z) \int d \mu_{y u}\left(r^{\prime}\right)=\Phi(x, z)(R y \mid u)$. In the same way one can show that the left-hand side converges to $\Phi(y, u)(R x \mid z)$. Therefore $\Phi(x, z)(R y \mid u)=$ $\Phi(y, u)(R x \mid z)$. Assuming that $(R y \mid u) \neq 0$ we obtain (7.6) with $\mu_{f}=\frac{\Phi(y, u)}{(R y \mid u}$. If $R^{\prime}$ is another positive selfadjoint operator acting on a Hilbert space $H^{\prime}$, then considering $R \oplus R^{\prime}$ acting on $H \oplus H^{\prime}$ one can easily show that the constant $\mu_{f}$ is the same for $R$ and $R^{\prime}$.

We continue the proof of Theorem 7.1. Notice that $R=\left(I-2 \rho^{2}\right)|R|$ and $f(R, \rho)$ may be considered as function of $|R|$ and $\rho$. We shall use Lemma 7.1 in each of the eigenspaces of $\rho$ separately. On $H(\rho=0), f(R, \rho)=f(|R|, 0)$, whereas on $H(\rho= \pm 1), f(R, \rho)=f(-|R|, \pm 1)$. Therefore we have three different functions of $|R|$ and consequently three $a$ priori different constants $\mu$ depending on the eigenvalue of $\rho$. In other words $\mu=\mu(\rho)$ is an operator being a function of $\rho$. Incorporating the coefficient $2 i \sin \frac{\hbar}{2}$ and the operator $\operatorname{sign} R=\left(I-2 \rho^{2}\right)$ into $\mu(\rho)$ we may rewrite (7.6) in the following way:

$$
\lim _{\lambda \rightarrow 0}\left(x\left|\frac{f(\lambda R, \rho)-I}{\lambda}\right| z\right)=\frac{1}{2 i \sin \frac{\hbar}{2}}(R x \mid \mu(\rho) z) .
$$

Comparing this formula with (7.4) we see that

$$
(R x \mid \mu(\rho) f(S, \sigma) y)=(x \mid f(S, \sigma) T y)-(T x \mid f(S, \sigma) y)
$$


for any $x \in D(R) \cap D(T)$ and $y \in D(T)$. Rewriting this formula in the following way:

$$
\left(\left(T+\mu(\rho)^{*} R\right) x \mid f(S, \sigma) y\right)=(x \mid f(S, \sigma) T y)
$$

we obtain

$$
\left(T+\mu(\rho)^{*} R\right)^{*} f(S, \sigma) \supset f(S, \sigma) T
$$

and passing to the adjoint operators we get

$$
T+\mu(\rho)^{*} R \subset f(S, \sigma) T f(S, \sigma)^{*} .
$$

On the right-hand side of the above inclusion we have a selfadjoint operator. Therefore the operator $Q=T+\mu(\rho)^{*} R$ is symmetric and $f(S, \sigma) T f(S, \sigma)^{*}$ is a selfadjoint extension of $Q$. Remembering that $\rho$ commutes with $R$ we see that $\mu(\rho)^{*}=\mu(\rho)$. We know that $\operatorname{sign} S$ commutes with $R, S$ and $T$. Therefore it has to commute with $\mu(\rho)$ and $\mu(-\rho)=\mu(\rho)$, for sign $S$ anticommutes with $\rho$. Remembering that $\operatorname{sign} T$ anticommutes with $\rho$ we conclude that $\mu(\rho)(\operatorname{sign} T)=(\operatorname{sign} T) \mu(\rho)$. Using this result one can easily show that $T \multimap \mu(\rho) R$. Now we shall apply Statement (4) of Theorem 5.2 to the extension (7.11):

$$
f(S, \sigma) T f(S, \sigma)^{*}=F_{\hbar}\left(\mu(\rho) S, \sigma^{\prime}\right) T F_{\hbar}\left(\mu(\rho) S, \sigma^{\prime}\right)^{*},
$$

where $\sigma^{\prime}$ is a reflection operator such that $\sigma^{\prime 2}=\chi(\mu(\rho) S<0), \sigma^{\prime}$ commutes with $S$ and anticommutes with $T$. It shows that $F_{\hbar}\left(\mu(\rho) S, \sigma^{\prime}\right)^{*} f(S, \sigma)$ commutes with $T$. We know that $|T|^{i t}$ commutes with $\sigma$ and $\sigma^{\prime}$ and scales $S$ be a positive factor. Therefore

$$
F_{\hbar}\left(\lambda \mu(\rho) S, \sigma^{\prime}\right)^{*} f(\lambda S, \sigma)
$$

does not depend on $\lambda>0$.

The limit (7.4) is finite for $x$ and $y$ running over a dense subset of $H$. Therefore $f(\lambda R, \rho)$ converges weakly to $I$, when $\lambda \rightarrow 0$. The same holds for $f(\lambda S, \sigma)$, because $(S, \sigma)$ is unitarily equivalent to $(R, \rho)$. Using this fact one can easily show that (7.13) weakly converges to $I$, when $\lambda \rightarrow 0$. Remembering that (7.13) does not depend on $\lambda$ we see that $F_{\hbar}\left(\lambda \mu(\rho) S, \sigma^{\prime}\right)^{*} f(\lambda S, \sigma)=I$ and

$$
f(\lambda S, \sigma)=F_{\hbar}\left(\lambda \mu(\rho) S, \sigma^{\prime}\right)
$$

for any $\lambda>0$.

Let $x, z \in D(S)$. The above formula shows that

$$
2 i \sin \frac{\hbar}{2} \lim _{\lambda \rightarrow 0}\left(x\left|\frac{f(\lambda S, \sigma)-I}{\lambda}\right| z\right)=2 i \sin \frac{\hbar}{2} \lim _{\lambda \rightarrow 0}\left(x\left|\frac{F_{\hbar}\left(\lambda \mu(\rho) S, \sigma^{\prime}\right)-I}{\lambda}\right| z\right) .
$$

By (1.8), the limit on the right-hand side exists and equals $(x|\mu(\rho) S| z)$. To compute the left-hand side it is sufficient to use (7.9) with $R$ and $\rho$ replaced by $S$ and $\sigma$. It shows that the left-hand side equals $(S x \mid \mu(\sigma) z)$. Therefore $(x|\mu(\rho) S| z)=$ $(S x \mid \mu(\sigma) z)$ and $\mu(\rho)=\mu(\sigma)$. Using the matrix notation (cf. (6.7)) we obtain

$$
\left(\begin{array}{cccc}
\mu_{o} & 0 & 0 & 0 \\
0 & \mu_{o} & 0 & 0 \\
0 & 0 & \mu_{s} & \mu_{a} \\
0 & 0 & \mu_{a} & \mu_{s}
\end{array}\right)=\left(\begin{array}{cccc}
\mu_{o} & 0 & 0 & 0 \\
0 & \mu_{s} & 0 & \mu_{a} \\
0 & 0 & \mu_{o} & 0 \\
0 & \mu_{a} & 0 & \mu_{s}
\end{array}\right)
$$


where $\mu_{o}=\mu(0), \mu_{s}=\frac{1}{2}(\mu(1)+\mu(-1))$ and $\mu_{a}=\frac{1}{2}(\mu(1)-\mu(-1))$. It shows that $\mu(0)=\mu(1)=\mu(-1)$. Denoting this common value by $\mu$ we see that $\mu(\rho)=\mu(\sigma)=$ $\mu I$. Now the formula (7.14) shows that

$$
f(S, \sigma)=F_{\hbar}\left(\mu S, \sigma^{\prime}\right) .
$$

Selfadjointness of $\mu(\rho)$ implies that $\mu \in \mathbf{R}$. Remembering that $\mu(\rho)=\mu I$ we see that both sides of (7.11) respect the direct sum decomposition $H=H^{\prime} \oplus H_{-+}$, where $H^{\prime}=H_{++} \oplus H_{+-} \oplus H_{--}$. Restricting (7.11) to the subspace $H_{-+} \subset H$ we obtain: $T_{o}+\mu R_{o} \subset f\left(S_{o}, 0\right) T f\left(S_{o}, 0\right)^{*}$. It shows that $T_{o}+\mu R_{o}$ has a selfadjoint extension. Assume for the moment that $\mu<0$. Then $T_{o}$ and $\mu R_{o}$ are of different signs and by Theorem 5.2 the operator $T_{o}+\mu R_{o}$ has no selfadjoint extension. This contradiction shows that $\mu \geq 0$.

Now we know that $\left(\sigma^{\prime}\right)^{2}=\chi(\mu S<0)=\chi(S<0)$. Remembering that $\sigma^{\prime}$ commutes with $S$ and anticommutes with $T$ one can easily show that $\sigma^{\prime}$ is of the form:

$$
\sigma^{\prime}=\left(\begin{array}{cccc}
0 & 0 & 0 & 0 \\
0 & 0 & 0 & s \\
0 & 0 & 0 & 0 \\
0 & s^{*} & 0 & 0
\end{array}\right),
$$

where $s$ is a unitary operator acting on $H_{o}$ commuting with $S_{o}$ and $T_{o}$. Using (6.9), (6.7) and the above formula we may rewrite (7.15) in the following form:

$$
\begin{aligned}
\left(\begin{array}{cccc}
f_{o}\left(S_{+}\right) & 0 & 0 & 0 \\
0 & f_{s}\left(S_{o}\right) & 0 & f_{a}\left(S_{o}\right) \\
0 & 0 & f_{o}\left(S_{o}\right) & 0 \\
0 & f_{a}\left(S_{o}\right) & 0 & f_{s}\left(S_{o}\right)
\end{array}\right) \\
\quad=\left(\begin{array}{cccc}
F_{o}\left(\mu S_{+}\right) & 0 & 0 & 0 \\
0 & F_{s}\left(\mu S_{o}\right) & 0 & s F_{a}\left(\mu S_{o}\right) \\
0 & 0 & F_{o}\left(\mu S_{o}\right) & 0 \\
0 & s^{*} F_{a}\left(\mu S_{o}\right) & 0 & F_{s}\left(\mu S_{o}\right)
\end{array}\right)
\end{aligned}
$$

where

$$
\begin{array}{rlrl}
f_{o}(r)=f(r, 0), & F_{o}(r)=F_{\hbar}(r, 0), \\
f_{s}(r)=\frac{1}{2}[f(-r,-1)+f(-r, 1)], & & F_{s}(r)=\frac{1}{2}\left[F_{\hbar}(-r,-1)+F_{\hbar}(-r, 1)\right], \\
f_{a}(r)=\frac{1}{2}[f(-r,-1)-f(-r, 1)], & & F_{a}(r)=\frac{1}{2}\left[F_{\hbar}(-r,-1)-F_{\hbar}(-r, 1)\right]
\end{array}
$$

for any $r \in \mathbf{R}_{+}$. Remembering that the spectral measure of $S_{o}$ is equivalent to the Lebesgue measure on $\mathbf{R}_{+}$we get: $s= \pm 1$ and $f_{o}(r)=F_{o}(\mu r), f_{s}(r)=F_{s}(\mu r)$, $f_{a}(r)=s F_{a}(\mu r)$ for almost all $r \in \mathbf{R}_{+}$. One can easily verify that these relations are equivalent to (7.2). 


\section{Exponential Function and Affiliation Relation}

In this section we freely use many notions of the theory of non-unital $C^{*}$-algebras. In particular for any $C^{*}$-algebra $A, M(A)$ is the multiplier algebra. The natural topology on $M(A)$ is the topology of strict convergence. The set of all elements affiliated with $A$ will be denoted by $A^{\eta}$. We write $R \eta A$ instead of $R \in A^{\eta}$. These notions are described in [14]. This section is devoted to the proof of the following:

Theorem 8.1. Let $R$ and $\rho$ be selfadjoint operators acting on a Hilbert space $H$ and $A$ be a non-degenerate $C^{*}$-subalgebra of $B(H)$. Assume that $\rho^{2}=\chi(R \neq 0)$ and $R \rho=\rho R$. Then the following two statements are equivalent:

(1) For any $t \in \mathbf{R}$ we have $F_{\hbar}(t R, \rho \chi(t R<0)) \in M(A)$. Moreover the mapping

$$
\mathbf{R} \ni t \longmapsto F_{\hbar}(t R, \rho \chi(t R<0)) \in M(A),
$$

where $M(A)$ is considered with the strict topology, is continuous.

(2) Operators $R$ and $R \rho$ are affiliated with $A$.

Proof. We shall consider the following commutative $C^{*}$-algebra:

$$
B=\left\{f \in C_{\infty}(\mathbf{R} \times\{-1,1\}): f(0,-1)=f(0,1)\right\} .
$$

Clearly $B=C_{\infty}(\Lambda)$, where $\Lambda$ is the locally compact space obtained from $\mathbf{R} \times\{-1,1\}$ by gluing points $(0,-1)$ and $(0,1)$. Elements of $B^{\eta}$ are continuous functions $f$ on $\mathbf{R} \times\{-1,1\}$ satisfying the condition $f(0,-1)=f(0,1)$. If in addition $f$ is bounded, then $f \in M(B)$. Let $t \in \mathbf{R}$. For any $r \in \mathbf{R}$ and $s= \pm 1$ we set:

$$
\begin{gathered}
f_{1}(r, s)=r, \quad f_{2}(r, s)=r s, \\
F^{t}(r, s)=F_{\hbar}(t r, s \chi(t r<0)) .
\end{gathered}
$$

Then $f_{1}, f_{2} \eta B$. Using [14, Example 2, page 497] we see that $f_{1}, f_{2}$ generate $B$. The asymptotic behavior (1.8) easily implies that $F^{t} \in M(B)$. Clearly $F^{t}$ is unitary. Remembering, that any continuous function on a compact set is uniformly continuous one can show that $F^{t}$ converges almost uniformly to $F^{t_{o}}$, when $t \rightarrow t_{o}$. For bounded sets, the strict topology on $M\left(C_{\infty}(\Lambda)\right)$ coincides with that of almost uniform convergence. Therefore the mapping

$$
\mathbf{R} \ni t \longmapsto F^{t} \in M(B)
$$

is continuous and for any function $\varphi \in L^{1}(\mathbf{R})$ we may consider integral

$$
F^{\varphi}=\int_{\mathbf{R}} F^{t} \varphi(t) d t \in M(B) .
$$

Taking into account the asymptotic behavior (1.13) and (1.15) one can show, that $F^{\varphi}(r, s) \rightarrow 0$, when $r \rightarrow \pm \infty$. It means that $F^{\varphi} \in B$.

Due to $(1.8), \lim _{t \rightarrow 0} \frac{1}{t}\left(F^{t}(r, s)-1\right)=r$. It shows that functions $F^{t}(t \in \mathbf{R})$ separate points $(r, s)$ and $\left(r^{\prime}, s^{\prime}\right)$ with $r \neq r^{\prime}$. Furthermore according to (1.19), 
$F^{t}(r,-1) \neq F^{t}(r, 1)$ for $t r<0$. Therefore the family of $\left\{F^{t}: t \in \mathbf{R}\right\}$ separates points of $\Lambda$. The same is valid for the family $\left\{F^{\varphi}: \varphi \in L^{1}(\mathbf{R})\right\}$. Now, using the Stone-Weierstrass theorem (applied to the one point compactification of $\Lambda$ ) we conclude that

$$
\begin{gathered}
\text { The }{ }^{*} \text {-subalgebra of } B \\
\text { generated by } \\
\left\{F^{\varphi}: \varphi \in L^{1}(\mathbf{R})\right\} \text { is dense in } B .
\end{gathered}
$$

Now, let $R$ and $\rho$ be the operators satisfying the assumptions of the theorem. Then the mapping

$$
\pi: B \ni f \longrightarrow f(R, \rho) \in B(H)
$$

is a non-degenerate representation of $B$. Clearly $\pi\left(f_{1}\right)=R$ and $\pi\left(f_{2}\right)=R \rho$. If $R, R \rho \eta A$, then $\pi \in \operatorname{Mor}(B, A)$. In this case

$$
F_{\hbar}(t R, \rho \chi(t R<0))=\pi\left(F^{t}\right) \in M(A)
$$

and the continuity of (8.1) follows immediately from that of (8.2). We showed that Statement (1) follows from Statement (2). Conversely assume that Statement (1) holds. Then $\pi\left(F^{t}\right) \in M(A)$ for all $t \in \mathbf{R}$ and the mapping

$$
\mathbf{R} \ni t \rightarrow \pi\left(F^{t}\right) \in M(A)
$$

is continuous. Integrating over $t$ we obtain $\pi\left(F^{\varphi}\right) \in M(A)$ for any $\varphi \in L^{1}(\mathbf{R})$. By (8.3), $\pi(B) \subset M(A)$. We shall use once more the continuity of (8.4). It implies that $F^{\varphi}$ tends strictly to $F^{0}=I$ when $\varphi$ is nonnegative, $\int_{\mathbf{R}} \varphi(t) d t=1$ and the support of $\varphi$ shrinks to $0 \in \mathbf{R}$. Therefore $\pi(B)$ contains an approximate unit for $A$ and $\pi \in \operatorname{Mor}(B, A)$. Applying $\pi$ to $f_{1}, f_{2} \eta B$ we obtain $R, R \rho \eta A$.

\section{Appendix A. Properties of $W_{\theta}$}

This appendix is devoted to the proof of Lemma 1.1. To prove (1.21) we compute the following integral:

$$
\begin{aligned}
\int_{0}^{N} \log (1+a) \frac{d a}{a} & =\int_{0}^{1} \log (1+a) \frac{d a}{a}+\int_{1}^{N} \log (1+a) \frac{d a}{a} \\
& =\int_{0}^{1} \log (1+a) \frac{d a}{a}+\int_{1 / N}^{1} \log \left(1+a^{-1}\right) \frac{d a}{a} \\
& =\int_{0}^{1} \log (1+a) \frac{d a}{a}+\int_{1 / N}^{1} \log (1+a) \frac{d a}{a}-\int_{1 / N}^{1}(\log a) \frac{d a}{a} \\
& =2 \int_{0}^{1} \log (1+a) \frac{d a}{a}-\int_{0}^{1 / N} \log (1+a) \frac{d a}{a}-\left.\frac{1}{2}(\log a)^{2}\right|_{1 / N} ^{1} \\
& =2 \int_{0}^{1} \log (1+a) \frac{d a}{a}-\int_{0}^{1 / N} \log (1+a) \frac{d a}{a}+\frac{1}{2}(\log N)^{2}
\end{aligned}
$$


According to [8, formula 3.661],

$$
\int_{0}^{1} \log (1+a) \frac{d a}{a}=\frac{\pi^{2}}{12}
$$

Therefore

$$
\int_{0}^{N} \log (1+a) \frac{d a}{a}=\frac{\pi^{2}}{6}-\int_{0}^{1 / N} \log (1+a) \frac{d a}{a}+\frac{1}{2}(\log N)^{2} .
$$

Inserting $a^{-1}$ instead of $a$ in (1.20) we get

$$
W_{\theta}(x)=\int_{0}^{\infty} \log \left(1+a^{\theta}\right) \frac{d a}{\left(1+a e^{-x}\right) a} .
$$

Therefore

$$
\begin{aligned}
W_{\theta}(-x) & =\int_{0}^{\infty} \log \left(1+a^{\theta}\right) \frac{e^{-x} d a}{\left(a+e^{-x}\right) a} \\
& =\int_{0}^{\infty} \log \left(1+a^{\theta}\right)\left[\frac{1}{a}-\frac{1}{a+e^{-x}}\right] d a .
\end{aligned}
$$

Combining this formula with (1.20) we get

$$
\begin{aligned}
W_{\theta}(x)+W_{\theta}(-x) & =\lim _{N \rightarrow \infty}\left(\int_{0}^{N} \log \left(1+a^{\theta}\right) \frac{d a}{a}+\int_{0}^{N} \log \frac{1+a^{-\theta}}{1+a^{\theta}} \frac{d a}{a+e^{-x}}\right) \\
& =\lim _{N \rightarrow \infty}\left(\int_{0}^{N} \log \left(1+a^{\theta}\right) \frac{d a}{a}-\theta \int_{0}^{N}(\log a) \frac{d a}{a+e^{-x}}\right)
\end{aligned}
$$

Computing the first integral we replace $a^{\theta}$ by $a$ and then use (A.1):

$$
\begin{aligned}
\int_{0}^{N} \log \left(1+a^{\theta}\right) \frac{d a}{a} & =\frac{1}{\theta} \int_{0}^{N^{\theta}} \log (1+a) \frac{d a}{a} \\
& =\frac{\pi^{2}}{6 \theta}-\frac{1}{\theta} \int_{0}^{N^{-\theta}} \log (1+a) \frac{d a}{a}+\frac{\theta}{2}(\log N)^{2}
\end{aligned}
$$

On the other hand,

$$
\begin{aligned}
\int_{0}^{N}(\log a) \frac{d a}{a+e^{-x}} & =\int_{0}^{N e^{x}} \log \left(a e^{-x}\right) \frac{d a}{a+1} \\
& =-x \int_{0}^{N e^{x}} \frac{d a}{a+1}+\int_{0}^{N e^{x}}(\log a) \frac{d a}{a+1}
\end{aligned}
$$




$$
\begin{aligned}
= & -\left.x \log (1+a)\right|_{0} ^{N e^{x}}+\left.(\log a) \log (1+a)\right|_{0} ^{N e^{x}} \\
& -\int_{0}^{N e^{x}} \log (a+1) \frac{d a}{a} \\
= & -x \log \left(1+N e^{x}\right)+(x+\log N) \log \left(1+N e^{x}\right) \\
& -\int_{0}^{N e^{x}} \log (a+1) \frac{d a}{a} \\
= & (\log N) \log \left(1+N e^{x}\right)-\int_{0}^{N e^{x}} \log (a+1) \frac{d a}{a} .
\end{aligned}
$$

Using now (A.1) we get

$$
\begin{aligned}
\int_{0}^{N}(\log a) \frac{d a}{a+e^{-x}}= & (\log N) \log \left(1+N e^{x}\right)-\frac{\pi^{2}}{6} \\
& +\int_{0}^{e^{-x} / N} \log (1+a) \frac{d a}{a}-\frac{1}{2}(x+\log N)^{2} \\
= & \frac{1}{2}(\log N)^{2}-\frac{1}{2} x^{2}+(\log N) \log \left(1+\frac{e^{-x}}{N}\right)-\frac{\pi^{2}}{6} \\
& +\int_{0}^{e^{-x} / N} \log (1+a) \frac{d a}{a} .
\end{aligned}
$$

Inserting these data into our main formula we see that $W_{\theta}(x)+W_{\theta}\left(x^{-1}\right)$ coincides with

$$
\begin{aligned}
\lim _{N \rightarrow \infty} & \left(\left(\theta+\theta^{-1}\right) \frac{\pi^{2}}{6}-\frac{1}{\theta} \int_{0}^{N^{-\theta}} \log (1+a) \frac{d a}{a}+\frac{\theta x^{2}}{2}\right. \\
& \left.-\theta(\log N) \log \left(1+\frac{e^{-x}}{N}\right)-\theta \int_{0}^{e^{-x} / N} \log (1+a) \frac{d a}{a}\right)
\end{aligned}
$$

and (1.21) follows.

We shall prove (1.22). Replacing in (1.20) $\theta$ by $1 / \theta$ we get

$$
W_{1 / \theta}(x)=\int_{0}^{\infty} \log \left(1+a^{-1 / \theta}\right) \frac{d a}{a+e^{-x}} .
$$

Inserting $e^{-x} a^{\theta}$ instead of $a$ in and then integrating by parts we obtain

$$
\begin{aligned}
W_{1 / \theta}(x) & =\int_{\infty}^{0} \log \left(1+e^{x / \theta} a\right) \frac{d\left(a^{-\theta}\right)}{a^{-\theta}+1} \\
& =-\int_{\infty}^{0} \log \left(1+a^{-\theta}\right) \frac{d\left(e^{x / \theta} a\right)}{e^{x / \theta} a+1} \\
& =\int_{0}^{\infty} \log \left(1+a^{-\theta}\right) \frac{d a}{a+e^{-x / \theta}}=W_{\theta}(x / \theta) .
\end{aligned}
$$


The formula (1.23) follows immediately from the equation

$$
\frac{1}{a+e^{-i \pi-x}}-\frac{1}{a+e^{i \pi-x}}=2 \pi i \delta\left(a-e^{-x}\right) .
$$

Let $a>0, z \in \mathbf{C}$ and $\varphi=\arg z$. We have

$$
\begin{aligned}
|a+z|^{2}-\cos ^{2}\left(\frac{\varphi}{2}\right)(|z|+a)^{2}= & \sin ^{2}\left(\frac{\varphi}{2}\right) a^{2}+2\left(\cos \varphi-\cos ^{2}\left(\frac{\varphi}{2}\right)\right) a|z| \\
& +\sin ^{2}\left(\frac{\varphi}{2}\right)|z|^{2} \\
= & \sin ^{2}\left(\frac{\varphi}{2}\right)\left(a^{2}-2 a|z|+|z|^{2}\right) \geq 0 .
\end{aligned}
$$

Therefore $|a+z| \geq \cos \left(\frac{\varphi}{2}\right)(|z|+a)$. Setting $z=e^{-x}$ and replacing $a$ by $a^{-1}$ we get

$$
\left|\frac{1}{a^{-1}+e^{-x}}\right| \leq \frac{1}{\cos \left(\frac{1}{2} \Im x\right)} \frac{1}{a^{-1}+e^{-\Re x}} .
$$

Let $\mathcal{R}(x)$ be the function introduced by (1.24). Using the formula

$$
\int_{0}^{\infty} a^{n} \log \left(1+a^{-\theta}\right) d a=\frac{\pi}{(n+1) \sin \left(\frac{(n+1) \pi}{\theta}\right)}
$$

one can easily verify that

$$
e^{-2 x} \mathcal{R}(x)=\int_{0}^{\infty} a \log \left(1+a^{-\theta}\right) \frac{e^{x} d a}{e^{x}+a^{-1}} .
$$

Taking into account the above estimate we obtain

$$
\left|e^{-2 x} \mathcal{R}(x)\right| \leq \frac{1}{\cos \left(\frac{1}{2} \Im x\right)} \int_{0}^{\infty} a \log \left(1+a^{-\theta}\right) \frac{e^{\Re x} d a}{e^{\Re x}+a^{-1}} .
$$

Remembering that $\theta>2$ one can easily show that the function $a \log \left(1+a^{-\theta}\right)$ is integrable on $[0, \infty$ [ and using the dominated convergence theorem of Lebesque we obtain the proof of (1.25).

\section{Appendix B. Fourier Transform of $V_{\theta}$}

This appendix is devoted to the proof of (1.41). Denote the left-hand side of this formula by $\Phi(x)$. Using (1.37) and (1.36) one can easily show that the integrand have the following asymptotic behaviour:

$$
V_{\theta}\left(y-\frac{i \hbar}{2}-i \pi\right) e^{\frac{i y^{2}}{2 \hbar}} \approx \begin{cases}e^{\frac{i y^{2}}{2 \hbar}} & \text { for } \Re y \rightarrow-\infty \\ C_{1} e^{-\frac{\hbar+2 \pi}{2 \hbar} y} & \text { for } \Re y \rightarrow+\infty\end{cases}
$$

where the phase factor $C_{1}=C_{\theta} e^{i(\hbar+2 \pi)^{2} / 8 \hbar}$ and $\theta=\frac{2 \pi}{\hbar}$. To avoid the oscillating behaviour of the integrand in (1.41) when $y \rightarrow-\infty$, we deform the integration path replacing $\ell$ by $\ell_{1}$ shown on the following drawing: 


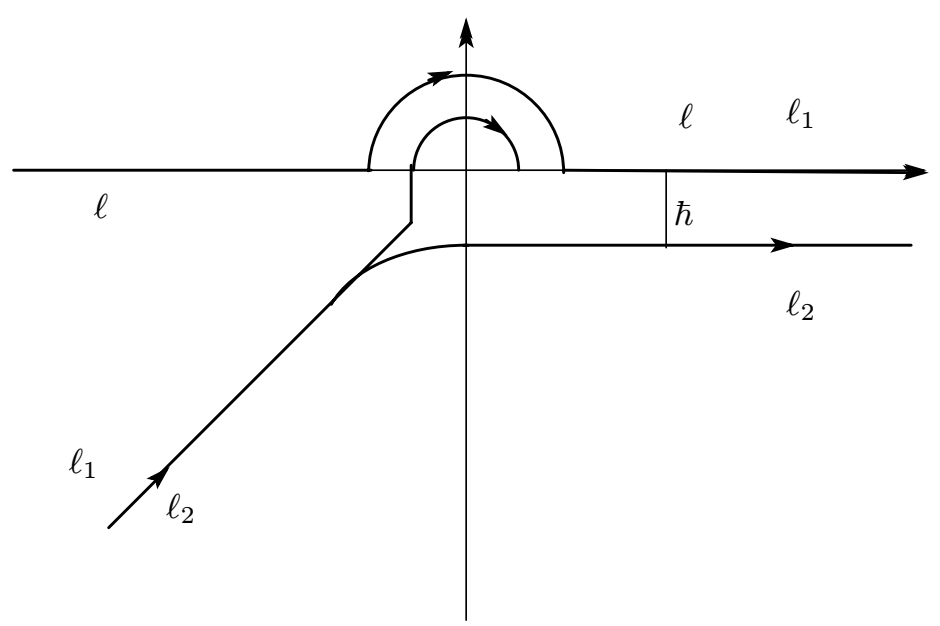

We have

$$
\Phi(x)=\frac{1}{\sqrt{2 \pi \hbar}} \int_{\ell_{1}} V_{\theta}\left(y-\frac{i \hbar}{2}-i \pi\right) e^{\frac{i y^{2}}{2 \hbar}} e^{\frac{i x y}{\hbar}} d y .
$$

This formula combined with (B.1) shows that $\Phi(x)$ is holomorphic in the region $\Im x>-\left(\frac{\hbar}{2}+\pi\right)$. We have to determine the asymptotic behaviour of $\Phi(x)$ when $\Re x \rightarrow \pm \infty$. By $(1.29)$

$$
V_{\theta}\left(y-\frac{i \hbar}{2}-i \pi\right)=\frac{V_{\theta}\left(y+\frac{i \hbar}{2}-i \pi\right)}{1-e^{y}} .
$$

It shows that the integrand in (B.2) has a pole at the point $y=0$ with the residuum equal to $-V_{\theta}\left(\frac{i \hbar}{2}-i \pi\right)$. Therefore

$$
\Phi(x)=i \sqrt{\frac{2 \pi}{\hbar}} V_{\theta}\left(\frac{i \hbar}{2}-i \pi\right)+\frac{1}{\sqrt{2 \pi \hbar}} \int_{\ell_{2}} V_{\theta}\left(y-\frac{i \hbar}{2}-i \pi\right) e^{\frac{i y^{2}}{2 \hbar}} e^{\frac{i x y}{\hbar}} d y,
$$

where the path $\ell_{2}$ is shown on the above drawing (due to (B.1) the integral over vertical line of length $\hbar$ connecting $\ell_{1}$ and $\ell_{2}$ tends to zero when the line moves horizontally to $+\infty)$. All points $y \in \ell_{2}$ have $\Im y \leq-\hbar$. If $y \in \ell_{2}, \Re x<0$ and $0 \leq \Im x \leq \hbar$ then

$$
\left|e^{\frac{i x y}{\hbar}}\right| \leq\left(1+e^{-\Re y}\right) e^{\Re x} .
$$

Using this estimate one can easily show that the integral on the right-hand side of (B.4) tends to 0 when $\Re x \rightarrow-\infty$. Therefore

$$
\Phi(x) \longrightarrow i \sqrt{\frac{2 \pi}{\hbar}} V_{\theta}\left(\frac{i \hbar}{2}-i \pi\right)
$$

when $\Re x \rightarrow-\infty$ and $0 \leq \Im x \leq \hbar$.

For $\Re x>0$ it is more convenient to investigate the function

$$
e^{\frac{i x^{2}}{2 \hbar}} \Phi(x)=\frac{1}{\sqrt{2 \pi \hbar}} \int_{\ell} V_{\theta}\left(y-\frac{i \hbar}{2}-i \pi\right) e^{\frac{i(x+y)^{2}}{2 \hbar}} d y .
$$


Using this formula and (1.37) one can show that

$$
e^{\frac{i x^{2}}{2 \hbar}} \Phi(x) \longrightarrow \frac{1}{\sqrt{2 \pi \hbar}} \int_{\mathbf{R}} e^{\frac{i y^{2}}{2 \hbar}} d y=\frac{1+i}{\sqrt{2}}
$$

when $\Re x \rightarrow+\infty$ and $0 \leq \Im x \leq \hbar$.

Remembering that the integrand in (B.2) is holomorphic everywhere above $\ell_{1}$ we obtain the same result integrating along the shifted path $\ell_{1}+i \hbar$ (due to (B.1) the integral over vertical line of length $\hbar$ connecting $\ell_{1}$ and $\ell_{1}+i \hbar$ tends to zero when the line moves horizontally to $\pm \infty$ ):

$$
\Phi(x)=\frac{1}{\sqrt{2 \pi \hbar}} \int_{\ell_{1}+i \hbar} V_{\theta}\left(y-\frac{i \hbar}{2}-i \pi\right) e^{\frac{i y^{2}}{2 \hbar}} e^{\frac{i x y}{\hbar}} d y .
$$

Inserting $y+i \hbar$ instead of $y$ we obtain

$$
e^{i \hbar / 2} e^{x} \Phi(x)=\frac{1}{\sqrt{2 \pi \hbar}} \int_{\ell_{1}} V_{\theta}\left(y+\frac{i \hbar}{2}-i \pi\right) e^{-y} e^{\frac{i y^{2}}{2 \hbar}} e^{\frac{i x y}{\hbar}} d y .
$$

Taking into account (B.3) we have

$$
\begin{aligned}
e^{i \hbar / 2} e^{x} \Phi(x) & =\frac{1}{\sqrt{2 \pi \hbar}} \int_{\ell_{1}} V_{\theta}\left(y-\frac{i \hbar}{2}-i \pi\right)\left(e^{-y}-1\right) e^{\frac{i y^{2}}{2 \hbar}} e^{\frac{i x y}{\hbar}} d y \\
& =\frac{1}{\sqrt{2 \pi \hbar}} \int_{\ell_{1}} V_{\theta}\left(y-\frac{i \hbar}{2}-i \pi\right) e^{\frac{i y^{2}}{2 \hbar}}\left(e^{\frac{i(x+i \hbar) y}{\hbar}}-e^{\frac{i x y}{\hbar}}\right) d y .
\end{aligned}
$$

Therefore

$$
e^{i \hbar / 2} e^{x} \Phi(x)=\Phi(x+i \hbar)-\Phi(x)
$$

and finally

$$
\Phi(x+i \hbar)=\left(1+e^{i \hbar / 2} e^{x}\right) \Phi(x) .
$$

We see that the function $\Phi$ satisfies the same functional equation as $V_{\theta}$ (cf. (1.31)). Therefore

$$
\frac{\Phi(x+i \hbar)}{V_{\theta}(x+i \hbar)}=\frac{\Phi(x)}{V_{\theta}(x)} .
$$

It means that $\frac{\Phi(x)}{V_{\theta}(x)}$ is a periodic function with the period $i \hbar$. Taking into account (1.37), (1.36), (B.5) and (B.7) one can easily show that this function is bounded in the strip $0 \leq \Im x \leq \hbar$. By periodicity it is a bounded holomorphic function on the whole complex plane. By Liouville theorem it is a constant function. Therefore $\Phi(x)=$ Const $V_{\theta}(x)$. To establish the value of the constant we compare (B.7) with (1.37) and (1.36). This way we obtain: Const $=\frac{1+i}{\sqrt{2}} C_{\theta}^{-1}$ and (1.41) follows.

\section{References}

[1] N. I. Achiezer and I. M. Glazman, Theory of Linear Operators in Hilbert Space, Pitman Publ., Boston, London, Melbourne, 1981.

[2] S. Baaj and G. Skandalis, "Unitaires multiplicative et dualité pour les produits croisés de $C^{*}$-algèbres", Ann. scient. Éc. Norm. Sup., $4^{e}$ série, t. 26 (1993) 425-488. 
[3] L. D. Faddeev, "Discrete Heisenberg - Weyl group and modular group", Lett. Math. Phys. 34 (1995) 249-254.

[4] P. R. Halmos, A Hilbert Space Problem Book, Berlin, Heidelberg, New York, Springer Verlag, 1974.

[5] R. M. Kashaev, The Lecture at the 7th International Colloquium on Quantum Groups and Integrable Systems, Prague, 1998.

[6] T. Kato, Perturbation Theory for Linear Operators, Springer Verlag, Berlin, Heidelberg, New York, 1966.

[7] K. Maurin, Methods of Hilbert Spaces, Warszawa, 1967.

[8] I. M. Ryshik and I. S. Gradstein, Tables of Series, Products and Integrals, Berlin, 1957.

[9] M. H. Stone, "Linear transformations in Hilbert space, III", in Operational Methods and Group Theory, Proc. National Acad. of Sciences of the USA 16 (1930).

[10] S. L. Woronowicz, "Operator systems and their application to the Tomita Takesaki theory", J. Operator Theory 2(2) (1979) 169-209.

[11] S. L. Woronowicz, "Operator equalities related to quantum E(2) group", Commun. Math. Phys., to appear.

[12] S. L. Woronowicz, "Quantum E(2)-group and its Pontryagin dual", Lett. Math. Phys. 23 (1991) 251-263.

[13] S. L. Woronowicz, "From multiplicative unitaries to quantum groups", Int. J. Math. 7(1) (1996) 127-149.

[14] S. L. Woronowicz, " $C^{*}$-algebras generated by unbounded elements", Rev. Math. Phys. $7(3)$ (1995) 481-521.

[15] S. L. Woronowicz and S. Zakrzewski, "Quantum " $a x+b$ " group", in preparation. 